\title{
Zu den Quellen des Fachvokabulars in der Olmützer Gerichtsordnung aus dem Jahre $1550^{1}$
}

\author{
Libuše SPÁČILOVÁ
}

\begin{abstract}
:
On terminological resources in the Court Regulations (1550) of Olomouc

The study presented in this article investigates the resources of legal terminology used in an important legal document written by the Olomouc court scribe Jindřich Polan. The first part of the study describes the development of German legal terminology in four stages, from the compilation of the 'Sachsenspiegel' until the beginning of the $19^{\text {th }}$ century. The second part deals with the characteristics of German legal terms in Polan's regulations, and the third part discusses the use of legal terminology of Latin origin in the same document. Polan's approach to Latin loanwords is also analyzed - especially his approach to their semanticization, which is intended to simplify users' understanding of the text and the morphology of loanwords.
\end{abstract}

Keywords: Court Regulations, Meissen Law Book, German legal terminology, Sachsenspiegel, legal document DOI: doi.org/10.15452/StudiaGermanistica.2020.27.0005

\section{Einleitung}

Die vorliegende Studie setzt sich zum Ziel, einen Beitrag zur Erforschung der Entwicklung der deutschen Rechtssprache zu leisten und einen kleinen Teil dieser Fachsprache zu skizzieren. Im Mittelpunkt stehen die Quellen des Rechtsvokabulars in der Olmützer Gerichtsordnung. Das frühneuhochdeutsche Rechtsdokument, dessen zeitgenössischer, ziemlich langer offizieller Titel Zu「ammengetragene artickel in form eines rechtlichen proces, wie diefelben von alters her bei dieser koniglichen stadt Olomuntz bei gerichte und auch in und vor gehegter bank in ubung gehalten, sambt andern nodturftigen underweifungen und zutreglichen vellen lautet (Bestand Archiv der Stadt Olmütz, im Weiteren AMO, Bücher, Sign. 116), verfasste Magister Heinrich Polan, ein bedeutender Olmützer Gerichts- und Schöpfenschreiber, im Jahre 1550. Die Anzahl der bis heute erhaltenen deutschen Manuskripte - insgesamt neun ${ }^{2}$ - zeugt von einer wichtigen Stelle dieser Gerichtsordnung

Der Beitrag entstand im Rahmen des Projekts Nr. 20-04393S „Jindřicha Polana Soudní ř́d z roku 1550. Př́spěvek k poznání magdeburského práva na severní Moravě [Heinrich Polans Gerichtsordnung aus dem Jahre 1550. Ein Beitrag zur Erkenntnis des Magdeburger Rechts in Nordmähren]“, das in den Jahren 2020-2022 von der tschechischen Forschungsagentur GA ČR finanziert wird.

2 Drei Manuskripte werden im Mährischen Landesarchiv Brno / Brünn (1599, 1612, 17. Jh.), zwei im Staatlichen Bezirksarchiv Olomouc / Olmütz $(1550,1583)$ und zwei im Landesarchiv Opava / Troppau (17. Jh., 1763) aufbewahrt. 
im Rechtsleben derjenigen mährischen Städte, die dem modifizierten sächsisch-magdeburgischen Recht unterlagen. Das Olmützer Manuskript diente auch als Vorlage für drei tschechische Übersetzungen, die erste entstand im Jahre 1562 für die Stadt Neutitschein (Nový Jičín), das Manuskript ist verschollen, und zwei weitere für Wallachisch Meseritsch (Valašské Meziř́íčí) - das Manuskript aus dem Jahre 1642 wird heute im Mährischen Landesarchiv Brünn (Brno) aufbewahrt und das Manuskript aus der Zeit zwischen den Jahren 1593-1651 befindet sich im Museum der Region Valašsko/Walachei in Wallachisch Meseritsch.

Im einleitenden Teil der vorliegenden Studie wird die Entwicklung der deutschen Rechtssprache skizziert, der zweite Teil widmet sich dem Rechtsvokabular in Polans Gerichtsordnung und im dritten Teil wird vor allem nach Quellen der Rechtstermini lateinischer Herkunft in diesem Rechtsdokument gesucht.

\section{Die Entwicklungsetappen der deutschen Rechtssprache}

Die deutsche Rechtssprache nimmt unter den Fachsprachen eine interessante Stellung ein. Einer der Gründe dafür besteht wahrscheinlich darin, dass sie mit der Jägersprache und der Seemannssprache zu den ältesten Fachsprachen überhaupt gehört (Schmidt-Wiegandt 1990:345). Was Umfang, Bedeutung und Reichtum der historischen Belege aus soziolinguistischer Sicht betrifft, kann mit ihr keine andere spezifische Sprache konkurrieren (Merk 1933:5). Die Besonderheit der Rechtssprache liegt darin, dass sie einerseits die Fachsprache der Juristen ist, die eine spezielle Ausbildung haben, andererseits sollte sie auch den Laien verständlich sein, denn sie steht in enger Beziehung zu anderen Fachsprachen, z. B. zur Handwerker- oder Kaufmannssprache (vgl. Schmidt-Wiegand 1998:281). Die Spuren dieser Sprache führen tief in voralthochdeutsche Zeit, in die Zeit der Völkerwanderung, zurück. Relativ früh bildete sich eine Rechtsterminologie heraus; von Anfang an war zudem die Formelhaftigkeit, die sich in Verwendung von Rechtsphraseologismen widerspiegelte, ein typisches Merkmal dieser Sprache (vgl. Schmid 2015:214). Bis heute dient die Rechtssprache nicht nur den Juristen, sondern allen, die mit dem Recht in Berührung kamen oder kommen, deshalb verdient sie die Bezeichnung der „Sprache des Rechtslebens“. Sie steht der Gemeinsprache nicht so fern, manche Linguisten hielten sie deshalb sogar für ein Subsystem der Gemeinsprache (Schmidt-Wiegand 1990:348).

Die erste Etappe der Entwicklung der deutschen Rechtssprache, in der sich die voralthochdeutschen und althochdeutschen Termini bildeten, dauerte von der Zeit der Völkerwanderung bis ins 13. Jahrhundert. In dieser Zeit wurde eine Reihe von Rechtstexten in lateinischer Sprache verfasst, die deutsche Rechtssprache blieb auf das Gebiet der mündlichen Kommunikation beschränkt.

Die ältesten Rechtsbegriffe stammen aus vorliterarischer Zeit, z. B. ahd., mhd. ban, heute Bann (9. Jh., in latinisierter Form im 7. Jh.; germ. *banna- ,Aufgebot, Befehl, Bann'), ahd. buoz(a), mhd. buoz, buoze, heute Buße (9. Jh.; germ. *botō ,Besserung'), ahd. mord, mhd. mort, heute Mord (9. Jh.; germ. *mur $\$ a$ - ,Mittel zum Sterben') oder ahd. mund, mhd. munt ,Schutz, Vormundschaft“ (9. Jh.; germ. *mundo ,Hand, Schutz'; die Bedeutung spiegelt sich bis heute im Wort Vormund wider). Es gibt auch alte Rechtswörter, die keltischer Herkunft sind, z. B. ahd. ambahti (8. Jh.), mhd. ambahte, heute Amt (aus kelt. *ambactos ,Höriger, Diener'), oder ahd. eid (8. Jh.), mhd. eit (aus air. oeth,Eid ${ }^{* 3}$ ). Der älteste Rechtswortschatz ist aus dem Lateinischen übernommen und ist in Stammesrechten der Langobarden, Franken, Bayern und weiterer Stämme zu finden (vgl. Schmid 2015:215). Da es jedoch für manche Sachverhalte keine lateinischen Äquivalente gab, wurden in lateinisch verfassten Stammesrechten volkssprachliche Begriffe verwendet, oft in latinisierter Form, die als Rechtswörter

Ein Manuskript ist im Archiv der Stadt Brno (Ende des 16. Jh.) und ein Manuskript im Staatlichen Bezirksarchiv Opava (1763) zu finden.

3 Das Lexem Eid, ein Ausdruck für die Selbstverfluchung bei Kelten und Germanen, geht auf die idg. Wurzel *ei, gehen') zurück und betont das Hervortreten bei der Eidesleistung (vgl. Schmidt-Wiegand 1977:56). Wolfgang Pfeifer und Rudolf E. Keller führen das Keltische als Ausgangssprache an (Pfeifer 1989:334; Keller 1995:119). Kluge hält dies für unwahrscheinlich (Kluge 2002:230). 
älter sind als der Rechtswortschatz in ahd. Rechtsquellen. Eine ergiebige Quelle des ahd. Rechtswortschatzes stellen Glossen dar; zur ältesten Schicht gehören beispielsweise die Rechtswörter in den sog. Malbergischen Glossen ${ }^{4}$ zur Lex Salica ${ }^{5}$ wie mallum ,Gericht' (zu ahd. mahal, mallare, vor Gericht laden', ahd. mahalen) oder sacebaro ,Sachverhalter' (zu ahd. sahha ,Sache' und ahd. beran ,tragen'). In den Canones-Glossen kann man beispielsweise Begriffe wie uuidarstab ,Rechtsstreit“ oder hantfestī ,Bekräftigung durch Handschlag‘ finden (vgl. Schmid 2015:215).

In der zweiten Entwicklungsetappe der deutschen Rechtssprache, die im 13. Jh. ihren Anfang hatte, wurde der mhd. und der mnd. Rechtswortschatz konstituiert. Reiches Material dafür bieten Urkunden, die als selbständige rechtsverbindliche Dokumente in deutscher Urkundensprache ausgefertigt wurden, und Gesetze, die in unterschiedlichen, dialektal gefärbten Geschäftssprachen verfasst sind. Das erste umfassende Rechtsbuch ${ }^{6}$ in deutscher Sprache, eine Übersetzung des lateinisch geschriebenen und zwischen den Jahren 1220 und 1235 ins Deutsche übertragenen Sachsenspiegels, bedeutete im deutschen Recht einen Übergang vom Mündlichen zum Schriftlichen. Dieses Rechtsbuch spielte besonders bei der Entstehung der Stadtrechte eine große Rolle. Wichtig für die weitere Entwicklung der Rechtsterminologie war vor allem der erste Teil des Sachsenspiegels, der dem Privat-, Straf- und Prozessrecht gewidmet war. Dieser Teil beeinflusste direkt elf von 27 damaligen Stadtrechten, insbesondere das Recht, das die Stadt Magdeburg und die von der Stadt gegründeten Dörfer benutzten. Das Magdeburger Recht, das 1241 durch den Magdeburger Ratserlass als ein selbstständiges Recht kodifiziert worden war, erwarb später eine große territoriale Reichweite und Bedeutung (Nowak 1965:233). Infolge der Ostkolonisation verbreitete sich dieses Recht in Ostmitteleuropa, wo Städte von deutschen Kolonisten gegründet wurden, denen das deutsche Recht, ius theutonicum, verliehen wurde. Dieser lateinische Terminus drückt eine Symbiose des Sachsenspiegels und des Magdeburger Rechts aus (MRB 350). Das deutsche Recht ließ eine ganze Reihe von Rechtsbüchern - Sammlungen des angewandten Rechts - entstehen; eines davon war das Meißner Rechtsbuch, das zwischen den Jahren 1357 und 1387 niedergeschrieben ${ }^{7}$ und in vielen Städten auf einem großen Territorium, z. B. in der Markgrafschaft Meißen, im Osterland ${ }^{8}$ und anderen Gebieten, aber auch in Schlesien und in nördlichen Teilen der böhmischen Länder, benutzt wurde (vgl. Spáčilová 2016:165).

Erst der Übergang vom Latein zur deutschen Sprache, die zeigte, dass sie die Rolle der Rechtssprache erfüllen kann, ermöglichte im 13. und 14. Jahrhundert eine intensive Entwicklung der Rechtsterminologie. Den Höhepunkt der zweiten Etappe stellten der Erlass der Bambergischen Halsgerichtsordnung im Jahre $1507^{9}$ und jener der Carolina von Kaiser Karl V. im Jahre 1532 dar (Merk 1933:20).

Am Beispiel des Meißner Rechtsbuchs, bei dessen Abfassung der Sachsenspiegel als wichtige Quelle benutzt wurde (Schmidt-Wiegand 1999:2342), können drei Gruppen von Rechtstermini vorgestellt werden (DRW I/1914:IX-X):

Die erste Gruppe bilden Rechtstermini im engeren Sinne, die ausschließlich in einem Rechtskontext verwendet wurden. Dazu gehören im Sachsenspiegel und im Meißner Rechtsbuch verwendete Ausdrücke wie hergewete ,bei Erbangelegenheiten: Sachen für den Gebrauch des Mannes,

$4 \quad$ Die Bezeichnung hängt mit den volkssprachlichen Wörtern im lateinischen Text (in) mallobergo ,auf dem Gerichtsberg‘ zusammen (Schmid 2015:215).

5 Die Lex Salica, die Gesetze der Salischen Franken, wurde um 800 ins Althochdeutsche übersetzt, heute sind nur noch Fragmente erhalten (Schmid 2015:215).

6 Rechtsbücher umfassen nicht Belege konkreter Rechtsakte (dazu dienten Urkunden oder Gedenkbücher), sondern Sammlungen von Rechtsvorschriften.

7 Das neben dem Sachsenspiegel bedeutendste und meistverbreitete Rechtsbuch, dessen Hauptquellen der Sachsenspiegel, das Zwickauer Stadtrecht und das Goslarer Stadtrecht waren (vgl. MRB, 50).

8 Osterland, lat. Orientalis plaga, Plisni / Plisnensis / Austrasia, war ein historisches Gebiet in Sachsen zwischen den Flüssen Saale und Mulde (vgl. Honzák/Neškudla/Pečenka/Stellner/Vlčková 1997:466).

9 Die Bambergische Halsgerichtsordnung ist vom großen Juristen jener Zeit, Landhofmeister Hans von Schwarzenberg, verfasst worden. 
auch Rüstung, Waffen, Heeresausrüstung', gerade ,Sachen zum persönlichen Gebrauch der Frau (Schmuck, Kleider, Stoffe, Küchengeräte) ${ }^{`}$, meistens im Sinne von ,Erbe' benutzt, morgengabe ,Geschenk des Mannes an die Frau am Morgen nach der Hochzeitsnacht; Mitgift", leibzucht ${ }^{10}$ ,Lebensunterhalt, Lebensunterhaltsquelle (vor allem für Witwen)', gehegete bank „Banngericht', notgewere ,Notwehr', aber auch die heute noch geläufigen Ausdrücke richter oder gericht. Ruth Schmidt-Wiegand zufolge bezeichnen solche Termini von vornherein eine rechtsspezifische Angelegenheit, und ein anderer Zusammenhang ist nicht denkbar (Schmidt-Wiegand 1999:2342).

Die zweite Gruppe stellen Rechtsausdrücke im weiteren Sinne dar, zu denen Wörter gehörten, die nicht nur in rechtlichen, sondern auch in anderen Kontexten gebraucht wurden, jedoch in einer rechtlichen Beziehung eine spezifische Bedeutung hatten, z. B. ausrichtung im Alltagsleben ,Zahlung, Ausrüstung; Bestellung', in der Rechtssprache ,gerichtliche Entscheidung, Schiedsspruch; Vorladung; Aussteuerung, Abfindung (der Kinder) ${ }^{‘}$; dreißigste in der Alltagssprache ,der dreißigste, Ordinalzahl', in der Rechtssprache ,Stichtag im Erbrecht; Tag der Testamentseröffnung'; antwort in der Alltagssprache ,Erwiderung', in der Rechtssprache ,Verteidigung des Beklagten'; ausrede im Alltagsleben ,das letzte entscheidende Wort', in der Rechtssprache ,Entschuldigung vor Gericht, gerichtliche Rechtfertigung', klagen in der Alltagssprache ,sich beschweren', in einem Rechtskontext auch ,beklagen', stuhl im Alltagsleben ,Sitzmöbel, Bank', im Rechtskontext ,Gerichtsstuhl, Gerichtsbank, Bank der Schöffen'.

Nichtrechtswörter, d. h. Ausdrücke aus dem Alltagsleben, die für das Recht aber relevant sind, gehören zur dritten Gruppe, z. B. ehefrau ,Gattin“; lämde, lemde ,Lähmung, lähmende Verletzung; körperliche Verletzung; Mangel; Verdamnis, Unmoral'; kebiskint ,uneheliches Kind'; swertmage ,Verwandter väterlicherseits'; mortliche slege ,mörderische Verletzungen'.

Was die Herkunft der Rechtstermini betrifft, kann man sowohl im Sachsenspiegel als auch im Meißner Rechtsbuch Erbwörter finden, z. B. ban, ding, erbe, schult u. a. Da der Rechtswortschatz eine Bindung an die mündliche Rechtstradition aufweist, kommen im deutschen Rechtswortschatz in dieser Entwicklungsetappe der Rechtstermini kaum Fremdwörter vor (vgl. Schmid 2015:224). An Lehnwörtern sind nur vereinzelt welche zu finden, die dem Alt- und Mittelhochdeutschen längst angepasst worden sind und sich zu Rechtsausdrücken entwickelten, z. B. kamph / kampf, lat. campus ,Zweikampf; Arten der Herausforderung zum gerichtlichen Zweikampf‘ oder zoll, lat. telōneum > toloneum ,Zoll‘ - beide sind Rechtsausdrücke im weiteren Sinne. Der Sachsenspiegel umfasst auch manche konservative Rechtstermini, die bereits während des Lebens Eikes von Repgow nicht mehr geläufig waren, die aber auch im Meißner Rechtsbuch vorkommen, da ihre Bedeutung erweitert oder aktualisiert werden konnte, z. B. handgemahl, Gut, durch das insbesondere bei den Schöffenbarfreien des Sachsenspiegels Schöffenfähigkeit begründet wird; das involvierte Gericht; Schöffenstuhl ${ }^{‘ 11}$ oder pflege ,Abgabe, Zins; Betreuung'.

In der zweiten Entwicklungsetappe der deutschen Rechtssprache kommen in Rechtsbüchern, Urkunden und Akten neben einzelnen Ausdrücken relativ oft auch juristische Phraseologismen vor, die zum festen Bestandteil der Rechtssprache wurden. Ein typisches Phänomen in mittelhochdeutschen und frühneuhochdeutschen Rechtstexten stellen zwei- oder mehrgliedrige Verbindungen dar, die ein Behelf für die Rechtssprache waren, um den vollen Umfang eines Begriffs durch Summierung ähnlicher oder identischer Bezeichnungen zu erschöpfen und juristisch unmissverständliche und einwandfreie Formulierungen zu erlangen (Hartweg/Wegera 2005:206).

In der Rechtssprache sind bereits seit althochdeutscher Zeit Zwillingsformeln beliebt, $\mathrm{d}$. $\mathrm{h}$. zweigliedrige Wortverbindungen, die aus Komponenten derselben Wortarten bestehen. Sie wurden wahrscheinlich bereits im langobardischen, angelsächsischen, teilweise auch im fränkischen und bayrischen Recht benutzt, ihren Ursprung sucht man in alten magischen und mythischen Vorstellungen (Dilcher 1961:67). Nicht unplausibel wirkt die Annahme einiger Historiolinguisten, dass am Anfang der Entstehung dieser „Prestigeformen“ (Habermann 2001:222) der Einfluss der antiken

Im Sachsenspiegel kommt nur der Terminus leibzucht vor; im Meißner Rechtsbuch daneben auch leibgeding.

1 Vgl. URL 3. 
Autoren (zitiert nach Besch 1964:202) gestanden habe. Weithin herrscht in der Forschung Einigkeit darüber, dass die Verwendung der Paarformeln in Anbetracht der Variation in den deutschen Sprachlandschaften auf einen erhöhten Grad von Verständlichkeit über die engere Sprachlandschaft des Schreibers hinaus zielte (Besch 1964: 203). In der Sprache des deutschen mittelalterlichen Rechts treffen lateinische und germanische Elemente zusammen. Ein wichtiges Vorbild stellte die antike Rhetorik und die Figur des Hendiadyoin ${ }^{12}$ dar; die Synonymenkoppelung war eine beliebte Technik bei der Bildung dieser Sprachmittel. Die Rhythmisierung der Rede, Wortwiederholung, Worthäufung und Alliteration sind magische Merkmale der Paarformeln (Matzinger-Pfister 1972:25), oft tritt noch eine übertragene Bedeutung der Wortverbindung hinzu. Obwohl in der Forschungsliteratur die Meinung zu finden ist, dass vollidiomatische Paarformeln vor dem Neuhochdeutschen praktisch nicht vorkommen (Jesko 2006:36), kann man in älteren Rechtstexten, beispielsweise im Meißner Rechtsbuch, Paarformeln finden, die teilidiomatisiert sind (czu haut vnd czu har, ${ }^{13}$ fol. 71va; Jar vnd tag, ${ }^{14}$ fol. 14ra; genge und gebe,${ }^{15}$ fol. 8vb; gewalt adir gewere, ${ }^{16}$ fol. 40va; haus vnd hof,,${ }^{17}$ fol. 18ra). Ein weiteres Merkmal dieser Phraseme sind die Häufigkeit des Vorkommens (die Paarformel jar vnd tag kommt beispielsweise im Meißner Rechtsbuch neunundzwanzigmal vor), die Alliteration ${ }^{18}$ (gewalt adir gewere, fol. 40va; notczoge adir notnunft, fol. 48vb; ledig vnd los, fol. 54rb) oder der Endreim (czu wegen czu stegen, fol. 3va); es gibt auch Belege, in denen zwei oder mehrere dieser Merkmale zu finden sind (an alle gedrank vnd getwank, fol. 1rb). Die Alliteration und der Endreim verstärken den Zusammenhang der Glieder (Schmidt-Wiegand 1984:1387). In den meisten Fällen sind solche Komponenten Substantive (rat noch tat, fol. 63rb), seltener Verben (wetten vnd bussen, fol. 24va), Adjektive (rechte los vnd erelos, fol. 47va; getrewlich vnd redelich, fol. 31 rb; vrey vnd ledig, fol. 62rb) oder Adverbien (mynner adir mer, fol. 69vb; výl adir wenýg, fol. 72rb).

Wenn wir die Paarformeln aus semantischer Sicht betrachten, finden wir nur selten welche, deren Komponenten echt synonym sind (genge vnd gebe, fol. 70rb; kraft vnd macht, fol. 13ra; land adir acker, fol. 30ra). Manche Glieder sind bedeutungsähnlich, viele sind aber nicht synonym, sie stellen vielmehr verschiedene Seiten ein und desselben Objekts oder Sachverhalts dar (haus vnd hof, fol. 18ra; gerade adir hergewette, fol. 7vb; mit leýbe und mit selen, fol. 42vb; lantrecht adir wychpylde, fol. 34vb; jar vnd tag, fol. 32vb; mit vinger vnd mit czungen, ${ }^{19}$ fol. 18ra). Auch komplementäre, im Gegensatz oder Kontrast zueinander stehende Verbindungen kommen im Meißner Rechtsbuch vor. Es handelt sich meistens um zwei Bestandteile ein und desselben Sachverhalts (tag vnd nacht, fol. 31va; lebende adir tot, fol. 32vb; arm vnd reych, fol. 31va). Dazu gehören auch die Phraseme, in deren Komponenten das Präfix vn-vorkommt (das recht adir vnrecht, ${ }^{20}$ fol. $17 \mathrm{vb}$; der geborne vnd der vngeborne, fol. 61vb). Man kann das Präfix vn- jedoch auch in beiden Gliedern finden (vnfuge vnd vngerichte, fol. 26vb); in solchen Verbindungen kann die Bedeutung der ersten Komponente die Bedeutung der zweiten verstärken, ergänzen oder erläutern (Matzinger-Pfister 1972:7).

12 ,Eins durch zwei` (Habermann 2001:198).

13 Es geht um den rechtssprachlichen mittelalterlichen Terminus für Leibesstrafen bzw. Züchtigungsstrafen, konkret handelte es sich um das Abschneiden der Haare und um Prügelstrafen. Diese Strafen wurden als milde Strafen verhängt, z. B. über schwangere Frauen, die eine Straftat begangen hatten; die lateinische Variante pro pelle et capillis wird seit dem 12. Jahrhundert benutzt (vgl. Jesko 2006:232; Köbler 2005:299).

14 Lat. annus et dies, eine häufige Zeitbestimmung unklarer Herkunft, die zum ersten Mal in Schriftstücken in den Jahren 769-775 verwendet wurde. Im 14. Jahrhundert wurde unter dieser Formel in Rechtssachen die belegte Frist von einem Jahr, sechs Wochen und drei Tagen verstanden (vgl. Köbler 2005:351; Jesko 2006:234).

15 Verwendet im Sinne, gängig, üblich, verbreitet‘ (vgl. Jesko 2006:162).

16 Dieser rechtssprachliche Terminus diente zur Bezeichnung von Besitz und Verfügungsgewalt (vgl. Jesko 2006:164).

17 Dieses Phrasem bezeichnet ,Immobilien und Grundbesitz‘ (vgl. Jesko 2006:231).

18 Ludwig Erich Schmitt zufolge könnten zweigliedrige Verbindungen, die sich durch die Alliteration auszeichnen, deutscher, nicht lateinischer Herkunft sein. Seiner Meinung nach ist die Alliteration typisch für die deutsche Rechtssprache (vgl. Schmitt 1936:95).

19 Das Phrasem wird im Zusammenhang mit dem Eid verwendet.

20 Das Phrasem bezeichnet die Gesamtheit der mit dem Gerichtswesen verbundenen Rechte, vgl. URL 2. 
In der dritten Etappe der Entwicklung dieser Fachsprache - in der Etappe der frühneuhochdeutschen Rechtssprache im Zeitraum vom 14. bis 16. Jh. - entstanden neue Textsorten; neben Stadtund Landrechten wurden Testamente, Protokolle von Ratssitzungen, Verhörprotokolle, ${ }^{21}$ Urfehden, ${ }^{22}$ Verträge, Zunftordnungen und weitere Arten juristischer Texte verfasst (vgl. Schmid 2015:230), daneben auch lateinisch-deutsche Vokabularien, z. B. im Jahre 1564 Leonhard Schwartzenbachs Synonyma (Hass-Zumkehr 1986). Diese Etappe dauert bis zum Eintritt in die Phase der Rezeption des römischen Rechts, die für die Blütezeit dieses Funktiolekts gehalten wird. Mit ihr hielt wieder die lateinische Sprache in die deutsche Rechtssprache Einzug - in Form lateinischer Rechtstermini. Zur Aufnahme und Anpassung des römischen Rechts sowie zur aktiven Arbeit mit diesem Recht kam es im Humanismus (vgl. Malý 1995:44); an den von 1348 an zahlreich gegründeten Universitäten wurde das römische Recht vorgetragen. Während die erste Phase der Rezeption im Hochmittelalter überwiegend theoretisch blieb (vgl. Dinzelbacher 1992:700), wurden im 15. Jahrhundert Normen des römischen Rechts zur Grundlage der Jurisdiktion; von den zentralen weltlichen Gerichten wurden sie immer mehr übernommen (vgl. Mitteis 1992:327). In den böhmischen Ländern wurde vor allem das Stadtrecht vom römischen Recht beeinflusst (vgl. Malý 1995:44). An der Prager Universität entstand im Jahre 1372 die juristische Fakultät, an der auch das römische Recht gelehrt wurde; nach der Schließung der juristischen Fakultät im Jahre 1419 wurde das römische Recht an der philosophischen Fakultät unterrichtet (Malý 1995:45).

In Mitteleuropa wurde die Aufnahme des römischen Rechts von der Entstehung juristischer Literatur begleitet. Neben mittelalterlichen alphabetischen Rechtsenzyklopädien erschienen gegen Ende des Mittelalters erste einfache Darstellungen des Prozessrechts. Zu den bedeutendsten Strafgerichtsordnungen gehört sicherlich die Bambergische Halsgerichtsordnung von 1507, die die Grundlage der seit 1532 für alle Reichsbestandteile geltenden Peinlichen Gerichtsordnung Kaiser Karls V. (Carolina) bildete.

Auf dem Territorium des Sächsischen bzw. Magdeburger Rechts wurde im 16. Jahrhundert eine bedeutende Gerichtsordnung von dem Juristen Kilian König verfasst, der im Jahre 1507 in Zwickau an der Bearbeitung der städtischen Statuten beteiligt war. Erst im Jahre 1541, 15 Jahre nach dem Tod des Verfassers, wurde Königs Prozesshandbuch unter zwei verschiedenen Titeln (Ein fast sehr und auserlesener Proce $\beta$, Practica ${ }^{23}$ und Gerichtsordnung und Processus und Practica der Gerichtsleuffte) in Leipzig gedruckt; doch dürfte es schon früher handschriftlich verbreitet gewesen sein. Königs Werk übte sicher einen entscheidenden Einfluss auf die sächsische Rechtspraxis aus. In den Gerichtsordnungen wurde im Allgemeinen das Fachwissen auf drei Ebenen des Gerichtsprozesses präsentiert: Die erste Ebene stellte die Gerichtspersonen und die Institution Gericht vor, die zweite behandelte den gesamten Rechtsprozess und die dritte charakterisierte einzelne Bestandteile des Gerichtsverfahrens.

Wahrscheinlich nach dem Vorbild von Königs Practica entstand in der Stadt Olmütz ein anderes bedeutendes Exemplar der Textsorte Gerichtsordnung. Das Titelblatt trägt neben dem langen, bereits im einleitenden Teil dieser Studie angeführten Titel Zusammengetragene artickel ... die Jahreszahl 1550. Verfasser dieser Ordnung war der aus Danzig stammende Heinrich Polan.

Die vierte Etappe der Entwicklung der deutschen Rechtssprache stellt die Zeit der großen Rechtskodifikation am Ende des 18. Jahrhunderts und am Anfang des 19. Jahrhunderts dar (SchmidtWiegand 1990: 348-349; Schmidt-Wiegand 1998:277).

${ }^{21}$ Dieser Oberbegriff ist weiter zu differenzieren, es gab verschiedene Subklassen, z. B. Urgichten. Die ursprüngliche Bedeutung des Wortes Urgicht, ahd. irgehan, mhd. erjehan, war ,Aussage“ oder ,Bekenntnis‘. Nach Grimms Wörterbuch erwarb das Wort mit der Einführung des römischen Rechts und der Folterung eine verengte Bedeutung und bezeichnete entweder ,Geständnis mit oder ohne Tortur' oder ,Aufzeichnung des Geständnisses‘. In diesem Beitrag wird der Begriff im Sinne von ,eine summarische Beschreibung aller Verbrechen eines Deliquenten` gebraucht.

22 Als Urfehden bezeichnete man Eide verwiesener oder entlassener Verhafteten, sich nicht zu rächen (vgl. Spáčilová 2009). Im Sachsenspiegel findet man die Bezeichnung urfehde (SSp, Buch I, Art. 8). Mehr über Urfehden und deren Untersuchung in Deutschland und in der Stadtkanzlei Olmütz vgl. Spáčilová 2010.

23 Practik, Pl. Practica,Art der Ausübung einer Tätigkeit, Verfahrensweise, Handhabung‘. 
Wie jede Fachsprache hat auch die Rechtssprache ihre Spezifika. Die Bemühung um eine präzise Ausdrucksweise führt zur Verwendung von Paarformeln, mehrgliedrigen Wortketten und Attributen an Stelle von Attributsätzen. Typisch ist eine Fülle von Synonymen für ein und denselben Begriff, die mit der Tendenz zu fachsprachlich bedingter Eindeutigkeit zusammenhängt (Schmidt-Wiegand 1998:281); sehr oft weisen Synonyme landschaftliche Unterschiede auf (vgl. DRW I/1914:XV; Schmidt-Wiegand 1998:281).

Nach diesen Vorbemerkungen kann das Lexikon der Olmützer Gerichtsordnung untersucht werden. Infolge der Rezeption des römischen Rechts und infolge der Tatsache, dass der Autor ein akademisch ausgebildeter Mann war, sind neben den angeführten Merkmalen auch das Vorkommen lateinischer Rechtsbegriffe zu erwarten.

\section{Die Olmützer Gerichtsordnung und ihr Verfasser Heinrich Polan}

Magister Heinrich Polan, der wahrscheinlich nach dem Jahre 1500 in Danzig geboren wurde ${ }^{24}$ war einer der bedeutendsten Stadtschreiber in Olmütz. In Danzig dürfte Heinrich seine ersten Kenntnisse in einer lateinischen Schule gewonnen haben (Zukal 1927:100), im Jahre 1522 studierte er wahrscheinlich an der Universität im polnischen Krakau, wo er sich vermutlich in den Bereichen Jura und klassische Sprachen ausbilden ließ. Immatrikuliert wurde er - unter der Voraussetzung, dass sein Vater Klemens hieß (Štěpán 2000:247; Spáčil 2001:305) - im Jahre 1522. ${ }^{25}$ Sein Studium an der Krakauer Universität musste er möglicherweise in Folge der Reformation unterbrechen. Polans weitere Lebensschritte sind uns bis 1540 nicht bekannt. Um das Jahr 1540 lebte er in der schlesischen Stadt Neiße, ${ }^{26}$ fünf Jahre später - 1545 - siedelte er nach Olmütz über (vgl. Spáčil 2001:305). ${ }^{27} \mathrm{Er}$ war in der Olmützer Stadtkanzlei als notarius publicus, d. h. öffentlicher Schreiber, tätig, im Jahre 1551 wurde er Gerichtsschreiber und nannte sich selbst Gerichtsschreiber und Schöpfenschreiber (notarius scabinorum). ${ }^{28}$ Im Jahre 1556 zog Polan nach Troppau um.

Da die Olmützer Gerichtsordnung an das Magdeburger Recht anknüpft und die Rezeption des römischen Rechts widerspiegelt, kann davon ausgegangen werden, dass lateinische Rechtstermini neben alten deutschen Rechtswörtern und Paarformeln reich vertreten sind.

Auch in der Gerichtsordnung kann der Rechtswortschatz nach den drei oben genannten Typen von Rechtsausdrücken unterschieden werden (DRW I/1914:IX-X). Verwendet wurden Rechtswörter im engeren Sinne, d. h. Ausdrücke, die ausschließlich in einem rechtlichen Kontext verwendet werden, z. B. caution, citacion, gehegte banckh, nochtaidingkh, procurator, richter, vorladunge und weitere. Daneben kommen auch Rechtswörter im weiteren Sinne vor, d. h. Ausdrücke, die auch in anderer Beziehung gebraucht werden, die jedoch in einer rechtlichen Beziehung einen besonderen Sinn haben, z. B. hof, klaeger, klage, part, sperrung, tat, burgschafft, geloben und andere. Einen wichtigen Teil des Vokabulars bilden Nichtrechtswörter, d. h. Ausdrücke, die zwar ein außerrechtliches Verhältnis bezeichnen, für den analysierten Text jedoch große Bedeutung haben, beispielsweise hand, finger, frage, haus. Ein wichtiges Kriterium für eine weitere Gliederung

24 Im Jahre 1557 verfasste Heinrich Polan als Stadtschreiber in Troppau den sog. Ratesspiegel. Auf Folio 3a führte er an: Derhalbenn sie [der Bürgermeister und die Ratsherren] auch dieses buch, denn Rathesspiegel genandt, mir, henrico polann vonn Danczik, dieser Zeitt stadtschreiber zusamme zu leßenn vnnd zu ewiger gedechtnus Inenn vnndt Irenn nochkommlichenn Rathmannenn zu beschreibenn beuehlenn [Archiv der Stadt Troppau, Bücher, Sign. 221, fol. 3r].

25 Auf Folio 98 der Krakauer Universitätsmatrikel wurde Henricus Clementis de Gdano dioc. Kvaviensis (Bistum Leslau) eingetragen (vgl. ASUC 2:214; auch Staehelin 1955:10).

26 Die einzige zu belegende Spur zu Polans Aufenthalt in Neiße bietet der folgende Eintrag in der Matrikel der Wiener Universität: Am 14. April im Jahre 1566 wurde Heinrichs ältester Sohn Heinrich als Heinricus Polanus a Polansdorff, Silesius Nisenus, immatrikuliert (MUNWU 1453-1630:208 - in der Matrikel auf fol. 88r). Dem Eintrag zufolge lebte die Familie Polan in dieser schlesischen Stadt.

27 Ende Juli 1545 war Polan in der Olmützer Stadtkanzlei tätig (vgl. Spáčil 2001:305).

28 Ein Eintrag vom 17. Juni 1551 informiert, dass der Bürgermeister und alle drei Räte entschieden, Heinrich Polan aus Neiße zum Schöffenschreiber und Gerichtsschreiber zu ernennen (vgl. AMO, Bücher, Sign. 2, fol. 19v-20v). Daneben bekleidete er das Amt des Blutschreibers (vgl. Spáčil 2001:44). 
des Rechtswortschatzes ist die Herkunft, deshalb werden im Weiteren deutsche Rechtstermini und Rechtstermini fremder (lateinischer) Herkunft getrennt behandelt.

\section{Deutsche Rechtstermini in Polans Gerichtsordnung}

Deutsche Rechtswörter, die als Erbwörter bezeichnet werden können, bilden den Kern des Rechtsvokabulars in der Gerichtsordnung. In der Gerichtsordnung finden wir Termini aus dem Bereich des ,peinlichen Gerichtswesens“, unter denen Rechtswörter im engeren Sinne wie gehegt ding (fol. 34v), gehegt recht (fol. 34v), gericht (fol. 74v), fronbote (fol. 6v, im Sachsenspiegel vronebode, im Meißner Rechtsbuch fronebote), pusse (fol. 6v, im Sachsenspiegel bute, buoz, buze, im Meißner Rechtsbuch busse), pahnteiding (fol. 35v) oder richter (fol. 2r) vertreten sind, die beispielsweise auch im alten Olmützer Rechtskodex Wenzels von Iglau aus den Jahren 1430-1492 vorkommen (vgl. AMO, Bücher, Sign. 1540, fol. 73ra, 74rb, 94va, 147ra, 249 rb u. a.). Dies kann ein Grund dafür sein, dass Polan diese Termini gar nicht erklärte. Neben diesen bereits bekannten Ausdrücken wurden im „Olmützer peinlichen Gerichtswesen“ Termini benutzt, die in den alten Rechtsbüchern des sächsisch-magdeburgischen Rechts in Olmütz nicht verwendet wurden. Heinrich Polan dürfte bezweifelt haben, dass sie allgemein bekannt sind, und deshalb erklärt er ihre Bedeutung. Er fragt z. B.: Was banrecht aigentliche haiffe und semantisiert das Kompositum dadurch, dass er zunächst die erste, eher unbekannte Komponente erläutert: Ban haift zu Sach「lenrecht fo viele als gewaldt, ein gerichte ader geding zu hegenn, vnder banne haift lo viele als vndter gehegtem Dinge. Dorumb haift banrecht, aigentlichen dorfon zu redenn fo viele als gehegtt rechtt (fol. 34v).

Polan semantisiert aber auch ganze Termini, deren Unkenntnis wahrscheinlich Probleme bereiten konnte:

Wer ein anwaldt ader machtman genanndt wirt

Vnd der yft ein machtman genandt, der frembde hendel aus beuehl feines hern vnd principal guttwilligklichenn zu handeln auf fich nimbtt. Dan niemandts magkh wider Seinenn willenn zum machtmanne ader anwalden gefaczt ader dorczu gedrungen werden (fol. 41r).

Nicht alle Semantisierungen sind so ausführlich. Den Terminus nachteiding/nochteiding erklärt Polan beispielsweise kurz und bündig auf folgende Weise: Es werden alhie zu Olomuncz alle Jhar drey banrecht gehaldtenn vnd das vierde ein nochtaidingkh (fol. $34 \mathrm{v}$ ).

Aus inhaltlicher Sicht kann man deutsche Rechtstermini in der Olmützer Gerichtsordnung in drei Wortfelder gliedern. Das erste Wortfeld umfasst Fachausdrücke, die verschiedene Rechtshandlungen während des Rechtsprozesses bezeichnen, z. B. die Rechtsbegriffe im engeren Sinne lauperungk (fol. 36v, ,Parteienanzeige'), endvrteil und beyurteil (fol. 64rv, ,Schlussurteil' und ,Zwischenspruch'), vorladunge (fol. 4r), gerichtszwang (fol. 4r, ,Gerichtsgewalt; Gerichtsbarkeit'), bannrecht (fol. 34v, ,Gerichtsbarkeit; die für ein bestimmtes Gebiet geltende, an festgesetzten Tagen gebotene Gerichtssammlung, Banngericht') und die Rechtsbegriffe im weiteren Sinne wie clage (fol. 6r).

Zum zweiten Wortfeld gehören Termini, die mit Gerichtsprozessen zusammenhängen, wie Bezeichnungen von Gebühren, Sanktionen, Beweismitteln, Schriftstücken und deren Bestandteilen und weiteren Objekten oder Gegenständen, die mit dem Verlauf des Rechtsprozesses zusammenhängen - Rechtsbegriffe im engeren Sinne wie wergeld (fol. 53v), erbgeld (fol. 23r), scheppenbuch (fol. 72r) oder gerichtssigille (fol. 42r) sind bereits in den alten Olmützer Rechtskodexen zu finden. Neu erscheinen Ausdrücke, die infolge der Vervollkommnung der Rechtsverhandlungen entstanden sind. Unter ihnen gibt es auch Begriffe, die nach Polans Meinung falsch verstanden werden könnten, und deswegen erklärt er ausführlich ihre Bedeutung, wie z. B. im Fall des Ausdrucks Spaehnen, eines Rechtsbegriffs im weiteren Sinne: Spaehnen, das ift ehr fchneidet einen Spahn aus der tueren ader tuergericht vnd gibt In dem klaeger. Solche Spaenunge Lal der gerichtsfchreiber ins Gerichts buch verczaichnen zu ewiger gedaechtnis, dorumb nimbtt ehr 1 gr. von dem Spaengeltt (fol. 24r). 
Weitere Beispiele für diese Kategorie sind frift ${ }^{29}$ (frift bei Gerichte zur beczalunge, fol. 6r) oder außruffung ${ }^{30}$ (außruffunge der heußer ader gruende, fol. 24r) und viele weitere mehr.

Das dritte Wortfeld umfasst deutsche Bezeichnungen von Personen, die am Gerichtsprozess beteiligt sind. Solche Ausdrücke gehören zu Rechtstermini im engeren Sinne. Die Personen werden entweder mit einem allgemeineren Begriff bezeichnet, z. B. gerichtsftande (fol. 64v), recht ficzer (fol. 61v), oder mit einem speziellen Ausdruck benannt, beispielsweise richter (fol. 64v), fronebote (fol. 81v), beklagter (fol. 69v), verteildter (fol. 69v, ,Verurteilter'), antworter (fol. 6r, ,Beklagter'), zeugenfuehrer (fol. 55r, wer zum Beweis seiner Sache Zeugen aufführt'), Icheppen אchreiber (fol. 36v), nochrychter (fol. 24r, ,Gerichtsbote, Büttel, Scherge; Scharfrichter, Henker') und andere. In der Gerichtsordnung erscheinen neben den Rechtsausdrücken im engeren und weiteren Sinne auch Wörter, die nicht direkt zum Rechtswesen gehörten, d. h. Ausdrücke des Alltagslebens, z. B. Iwertmage (fol. 54v, ,Verwandter von väterlicher Seite').

Bei deutschen Rechtsbegriffen stößt man oft auf Polysemie (vgl. Papsonová 2000). Sie ist auch in Polans Gerichtsordnung nachzuweisen. Beispielsweise hat das Wort recht mehrere Bedeutungen und wird in der Gerichtsordnung im Sinne von ,Gerichtsprozess' (Ausgangkh des Rechten, fol. 2r), ,Urteil' (mit vrteil vnnd rechte, fol. 71r), ,Gericht‘ (gehegt recht, fol. 34v), ,Pflicht' (ane weither recht vnd pfandt, fol. 28v) und ,Privilegium“ oder, Recht' (noch Stadtrechten, fol. 11v) verwendet. Ein anderes Beispiel stellt das Wort ding dar, das in der Olmützer Gerichtsordnung im Sinne von ,Angelegenheit' (ein loeblich dingkh, fol. 8v), ,Sache' (ein vbriges dingkh, fol. 39v) und ,Gericht" (vndter gehegtem ding, fol. 34v) benutzt wird.

\subsection{Deutsche Formeln}

Neben den selbständigen deutschen Rechtstermini kommen in der Gerichtsordnung Formeln mit Komponenten ausschließlich deutscher Herkunft vor. Die Zwei- und Mehrgliedrigkeit war ein wichtiges Hilfsmittel für die Rechtssprache, um den vollen Umfang eines Begriffs durch Summierung ähnlicher oder identischer Bezeichnungen zu erschöpfen und juristisch unmissverständliche und einwandfreie Formulierungen zu erlangen (vgl. Hartweg/Wegera 2005:206). Auch Heinrich Polan bedient sich dieses traditionellen Mittels.

\subsubsection{Formeln mit (teil-)synonymen Komponenten}

In der Gerichtsordnung werden Formeln verwendet, die aus synonymen Wortpaaren bestehen, mit dem Ziel, eine Aussage zu verstärken. Um echte Synonyme aus den verschiedenen Gruppen der Rechtswörter handelt es sich relativ selten: vertreten sind Einheiten aus der Gruppe der Rechtswörter im engeren Sinne, z. B. der Foyt ader Richter (fol. 9r), mit dem Fronebohtenn ader Gerichtsdiehner (fol. $12 \mathrm{v}$ ), aus dem thurme vnd gefengknis (fol. 28v), neben der billigkheit vnnd gerechtigkheit (fol. 12v) oder Solch verfiegelungkh vnnd Sperrunge (fol. 17r); dementsprechend auch verfiegelnn ader Sperren (fol. 17r); ein gerichte ader geding (fol. 34v); ein folcher vorftand vnd burgfchafft $t^{31}$ (fol. 48r); bey gerichte vnnd Rechte (fol. 14v); mehr macht vnnd gewaldtt (fol. 42v) oder beweyfunge ader zeugen $^{32}$ (fol. 55r). Aus der Gruppe der Rechtswörter im weiteren Sinne sind das beispielsweise die Fügungen bekweme Zeit vnnd Frift (fol. 7r) oder laupern vnd an agen ${ }^{33}$ (fol. 37r) und aus der Gruppe der Wörter, die nicht zum Rechtswesen gehören, aber im Bereich der Rechtssprache eine

29 Frist ,Sächsische Frist (ein Jahr, sechs Wochen, drei Tage), Aufschub, Verzögerung; Nachsicht der Pflicht zur Handarbeit in einer Zeche', vgl. URL 1 (diese Internetquelle wurde auch bei folgenden Belegen verwendet).

30 Ausrufung,Ausrufen (bei der öffentlichen Versteigerung)‘.

31 Burgfchafft, einen Vorstand haben, sich persönlich vor Gericht stellen müssen“.

32 Zeuge,Zeugenbeweis'.

33 Laupern (oder lautbaren), vorladen; aufrufen; melden, anmelden, öffentlich bekanntgeben '; ansagen , anmelden; befehlen; Urteil verkündigen; laden, vorladen, ankündigen, angeben'. 
bedeutende Aufgabe übernehmen können, z. B. zeitt vnnd weile kommen iᄃt (fol. 73v), oder ganz allgemeine synonyme Ausdrücke wie auffreihem ringe vnnd markte (fol. 24r), mit gun It vnd laube ${ }^{34}$ (fol. 36v) oder obs vnd fruechte (fol. 25r).

Am häufigsten werden Substantive gekoppelt, während gekoppelte Verben (gelden vnd betzalen, fol. 48r; Itille Itehenn vnd hafften, fol. 47r) und Adjektive / Adverbien (vnd Colcher proces ift nichtigk vnd nullus, fol. 4r) seltener sind. Das letzte Beispiel ist auch ein Beleg für die Alliteration, weiter z. B. Teine zugefuegte Ichmach vnnd Ichaden (fol. 49r).

Öfter als echte Synonyme erscheinen bedeutungsverwandte Substantive oder Verben, die mehrgliedrige Ketten bilden: keine furchte vnd gefahr (fol. 61v); lafter vnnd vbel (fol. 9r); die gewere ruert ader bricht (fol. 53v) oder zeugen einbrengenn ader furftellen (fol. 55r). Die Reihung gleicher Ausdrücke kommt jedoch nur selten vor, z. B. von worthe zu worthe (fol. 59r); von mainunge zu mainunge (fol. 59v). Diese Einheiten in zweigliedrigen Ketten in der Gerichtsordnung gehören zur Gemeinsprache. Das häufigste Verbindungselement einzelner Komponenten in den charakterisierten Formeln sind die Konjunktionen vnd und oder; die Konjunktion vnd stellt ein kopulatives, die Konjunktion ader ein disjunktives Verhältnis zwischen den einzelnen Elementen her.

\subsubsection{Formeln mit nicht synonymen Komponenten}

In Polans Gerichtsordnung kommen neben Formeln mit synonymen Komponenten auch Zwillingsformeln vor, die aus antonymen Einheiten bestehen, wobei diese Komponenten oft keine Rechtswörter darstellen. Es sind meistens Adjektive: junkh ader aldtt (fol. 58v) oder armen vnnd reichen (fol. $72 \mathrm{v}$ ). Häufig sind polare komplementäre Lexeme, deren Verhältnis sich als Entweder-oder-Beziehung benimmt, z. B. kegenwertigkh ${ }^{35}$ ader abwehCens (fol. 43r); von frembden vnd ainheimifchen (fol. 29v); gaiftlich ader weltlich, gemainlich ader fonderlich (fol. 28v); viele ader wenigkh (fol. 25v) oder das nayn vnnd jha (fol. 8v). Die Formeln mit Verben kommen vereinzelt vor: der bekennet nicht vnnd lauknet auch nicht (lauknen = leugnen; fol. 46r). Schließlich erscheinen komplementäre Lexeme als Wortbildungskonstruktionen mit dem Präfix $v n$ - in der Gerichtsordnung, beispielsweise gerecht ader vngerechtt (fol. 64v); gefchickt ader vngelchickt (fol. 45r); recht ader vnrecht (fol. 9r) oder bewegliche noch vnbewegliche guetter (fol. 70v). Zu Formeln aus nicht synonymen Komponenten gehören neben antonymen Koppelungen aufzählende Reihungen. Die Beziehungen zwischen den einzelnen Komponenten können verschieden sein. In den meisten Fällen handelt es sich um Aufzählungen von Personen, Sachverhalten oder Merkmalen, die thematisch irgendwie miteinander auf dem Gebiet der Rechtswörter im engeren oder weiteren Sinne zusammenhängen: nochrichter ader Gerichtsdiehner (fol. 36v); ein wergelt vnd die gerichts koften vnd Ichaeden (fol. 49v); in viele vnd groIfe vnkolt vnnd Ichaden (fol. 8v); vnkolten vnd nochtail (fol. 40r); vbeltat, mi؟letat vnd lafter (fol. 44r); offenbare lafter vnd vbel (fol. 9r); von Siegelung vnd Sperrung (fol. 17r); Sperren ader verfiegelnn (fol. 17r); auff gruende, heuโzer, geldtt, fahrende habe vndt gutt (fol. 32r) und viele andere.

Selten sind Aufzählungen von Ausdrücken, von denen der eine eher eine allgemeine, der andere eine spezielle Bedeutung hat: durch den gerichts diehner ader Con te bekweme perCone (fol. 15r; das Wort person wurde im Mhd. entlehnt und hatte sich in der deutschen Sprache des 16. Jh. höchstwahrscheinlich eingebürgert; vgl. Kluge 2002: 691-692); von Anwalden ader machtleuten (fol. 41r); ein anwalt ader machtman (fol. 41r).

Während die Formeln mit synonymen Komponenten eine Verstärkung oder Betonung der vermittelten Aussagen erzielen sollen, besteht die Aufgabe der Formeln mit nicht synonymen Komponenten in der Präzisierung von Informationen.

\footnotetext{
Laub ,Erlaubnis, Genehmigung‘.
}

Kegenwertigh ,anwesend‘. 


\subsubsection{Deutsche Rechtssprichwörter}

Mit der Pragmatik alter Rechtstexte hängen neben den Paarformeln auch Rechtssprichwörter zusammen. Ruth Schmidt-Wiegand zufolge ist das Rechtssprichwort eine Sonderform des Sprichworts, nämlich eines, dem ein bestimmter Rechtssatz zugrunde liegt. Im Rechtssatz lässt sich das mündlich tradierte Gewohnheitsrecht erkennen (Schmidt-Wiegand 2002:9-10). Bei manchen Rechtssprichwörtern kann man Parallelen in lateinischer Sprache finden, z. B. für das Rechtssprichwort Wer zuerst kommt, mahlt zuerst, das im Sachsenspiegel um $1230 \mathrm{zu}$ belegen ist, existiert eine ältere lateinische Form aus dem bayerischen Kloster Schäftlarn Qui capit ante molam, merito molit ante farinam (Schmidt-Wiegand 2002:13). Gerade dieses Rechtssprichwort kommt im Sachsenspiegel in folgender niederdeutscher Form vor, z. B. De ok erst to der molen kumt, de scal erst over gan (SSp, Landrecht, Buch 2, 59, §4, Wer zuerst zur Mühle kommt, soll zuerst mahlen.'). Auch andere Sprichwörter erscheinen im Sachsenspiegel, z. B. Men seget, dat nen kint siner muder keves kind ne si (SSp, Landrecht, Buch I, 51, §2, ,Kein Kind ist seiner Mutter Kebskind.'), weiter Herren unde mannes valsche rat geliket wol ungetruwer dat (SSp, Lehenrecht 76, § 6, ,Herren und Mannes falscher Rat gleicht wohl ungetreuer Tat.') oder De eldere scal delen unde der jungere scal kesen (SSp, Landrecht, Buch III, 29, §2, ,Der Ältere soll teilen, der Jüngere wählen. '), De sone ne antwardet vor den vader nicht (SSp, Landrecht, Buch 2, 17, §1, ,Der Sohn antwortet nicht für den Vater.'), Des mannes sat, de he mit sineme pluge werket, de is verdenet, alse de egede dar ober geit (SSp, Landrecht, Buch II, 58, §2, ,Des Mannes Saat ist verdient, sobald die Egge darüber fährt.').

Diese Rechtssprichwörter erscheinen auch im Meißner Rechtsbuch, wie folgende Belege zeigen: ... wer ee zu der mole kumpt, der melt auch ee (MRB, V. Buch, Kap. 29.2, ,Wer zuerst zur Mühle kommt, soll zuerst mahlen.'); ..., das keyn kynt seyner müter kebyskynt sey (MRB, I. Buch, Kap. 13.6, ,Kein Kind ist seiner Mutter Kebskind.'), Der eldeste sal teylen, der jungiste sal kysen (MRB, I. Buch, Kap. 6, die modifizierte Form des Sprichwortes ,Der Ältere soll teilen, der Jüngere wählen.'); Der son antwort vor den vater nicht (MRB, IV. Buch, Kap. 22.1, ,Der Sohn antwortet nicht für den Vater.'). Des mannes saat, dy her mit seyme phluge vordynet hat mit erbeyt, ist vordynet, wenn dy eyde das lant bewert (MRB, II. Buch, Kap. 5.1, ,Des Mannes Saat ist verdient, sobald die Egge darüber fährt.').

Es stellt sich die Frage, ob auch Heinrich Polan in seiner Gerichtsordnung Rechtssprichwörter benutzte, die - wie sich gerade gezeigt hat - zum alten Rechtswortschatz gehörten. Aus den alten Olmützer Quellen ist bekannt, dass Polan gern Sentenzen und Sprichwörter in die Olmützer Stadtbücher eintrug, und zwar sowohl in lateinischer als auch in deutscher Sprache, z. B. Sors omnia versat. - Zeyt bringt roßen (AMO, Sign. 1046, fol. 1r). Sogar in alttschechischer Sprache verfasste er folgende Sentenz Ve všem mém zarmúcení / pán buoh mé utěšení / mlčeti jest také umění (AMO, Bücher, Sign. 1048, fol. 1r) ${ }^{36}$ - ein Beweis für seine Tschechischkenntnisse. Polan benutzte in seiner Gerichtsordnung eine Aussage, die Rechtssprichwörtern ähnelt, beispielsweise ... das nayn vnnd Jha zum Rechtenn gehoert (fol. 8v), was uns an die Worte in der Bibel erinnert: Eure Rede aber sei: Ja, ja; nein, nein. Was darüber ist, das ist vom Übel (Die Bibel, Matthäus 5.37).

\section{Lateinische Rechtsbegriffe in der Olmützer Gerichtsordnung}

Das Vorkommen lateinischer Begriffe in frühneuhochdeutschen Rechtsquellen hängt mit dem Einfluss des römischen Rechts zusammen. Es ist bisher nicht ganz klar, wann die Anfänge der Rezeption des römischen Rechts im Olmützer Gerichtswesen zu suchen sind. Rechtstermini lateinischen Ursprungs tauchen in den Olmützer Stadtbüchern nicht mit gleicher Häufigkeit wie die deutschen auf. Während das Vorkommen solcher Wörter in den nichtspezialisierten Stadtbüchern aus dem 15. Jahrhundert selten ist und auch zwei ältere Testamentsbücher aus den Jahren 1511-1541 und 1541-1556 arm an lateinischen Ausdrücken sind, kommen lateinische Termini häufiger erst im dritten Olmützer Testamentsbuch aus den Jahren 1572-1623 und im Hinterlassenschaftsbuch aus

36 „In all meiner Betrübnis ist Gott mein Trost, das Schweigen ist auch die Kunst.“ [Übersetzt von Libuše Spáčilová.] 
den Jahren 1579-1585 vor. Diese Tatsache dürfte mit einer tiefgreifenden Rezeption des römischen Rechts nördlich der Alpen und auch mit der akademischen Ausbildung der Schreiber in der zweiten Hälfte des 16. Jh. zusammenhängen. Polan, der eine lateinische Schule in Danzig besuchte und an einer Universität studierte, verfügte ganz sicher über sehr gute Lateinkenntnisse. Das war die beste Voraussetzung für die Verwendung lateinischer Ausdrücke. Polan blieb dieser Voraussetzung nichts schuldig - das Vorkommen lateinischer Rechtsbegriffe ist eines der Hauptmerkmale des Lexikons dieser Gerichtsordnung.

In der Olmützer Gerichtsordnung befinden sich lateinische Ausdrücke, die ohne weitere deutsche Erläuterung benutzt werden. Polan dürfte vorausgesetzt haben, dass sie allgemein bekannt sind und Vögten und Schöffen, denen die Gerichtsordnung dienen sollte, keine Schwierigkeiten bereiten.

\subsection{Lateinische Termini für Personenbezeichnungen}

$\mathrm{Zu}$ solchen Termini gehören Bezeichnungen von Personen, die mit dem Recht etwas zu tun hatten. Bereits im Jahre 1424 erscheint im Olmützer Rechtskodex Wenzels von Iglau (AMO, Bücher, Sign. 1540, fol. 273ra) der Terminus procurator, deshalb konnte Heinrich Polan in seiner Gerichtsordnung voraussetzen, dass dieser Terminus keiner Erklärung bedarf. Er verwendet ihn relativ häufig, z. B. durch Ceinen procurator frogen (fol. 68r); Procurator des beklagten (fol. 52r). Interessant ist, wie Polan dieses Wort flektiert. Er empfindet den Ausdruck als Fremdwort, deshalb versieht er ihn mit lateinischen Flexionsendungen: nicht who die procuratores Itehenn (fol. 56v); beide mit yren procuratoribus erfcheinen (fol. 51r); einem jeden hern Foyte vnd Scheppen, Notarien, Advocaten, Procuratoribus (fol. I, Vorrede) oder mit yren procuratoribus (fol. 51r). Dass Polan dabei nicht konsequent vorgeht, belegen zwei weitere Beispiele: die zwehne gefchworne procurator (fol. 36v) und den zwehen gefchwornen Procuratoren (fol. 35r). Dieses Wort ist nicht der einzige Ausdruck mit lateinischen Endungen, so finden wir z. B. die Verbindung der meifte hauffen der doctores dorwider (fol. 66r), eine andere sogar mit einer lateinischen Präposition der ltriedt Inter doctoribus (fol. 65v). Interessant ist die Verwendung des Begriffs principal (fol. 47v), der auch in seiner movierten Form mit dem Movierungssuffix -in vorkommt: die fraue als die principalin (fol. 54v, ,Bevollmächtigte“, Sommer 1833:389).

$\mathrm{Zu}$ den lateinischen Rechtstermini, die ohne Erklärung benutzt werden, gehört auch die Bezeichnung klienten (fol. 40r), die an anderen Stellen des Dokuments durch den Ausdruck parten ersetzt wurde - kain part angefehen (fol. 21r) und die parthen, die zu dem rechten verburgt lein (fol. 36v).

\subsection{Lateinische Termini für Rechtshandlungen während des Rechtsprozesses}

Die meisten lateinischen Termini bezeichnen Rechtshandlungen oder deren Teile. Die ganze Verhandlung vor Gericht wird in der Gerichtsordnung als procels bezeichnet - noch folchem procels (fol. 58v), betont wird fundament des prozes (fol. 4r), d. h. Grundlage der Rechtshandlung. Verhandlungen vor Gericht nennt Polan auch acten - mit diefen acten abfertigenn wolden (fol. 61v). Lateinisch werden u. a. Gründe, die den Prozess veranlassen, benannt: Iubornation als heimliche Verführung zum Bösen (fol. 61v) und Iniurien Cachen als Ehrverletzungen (fol. 9v). Als Citirung für gerichte wird die Vorladung vor Gericht bezeichnet (fol. 72r).

Auch einzelne Rechtsgeschäfte oder mündliche bzw. schriftliche Produkte des Rechtsprozesses werden in der Gerichtsordnung lateinisch bezeichnet. $\mathrm{Zu}$ diesen Teilhandlungen gehören Exception wider die Zeugen (fol. 58r, 61v, ,Gegenrede', vgl. Prätorius 1875:108), Des Zeugenfuehrers defenfion (fol. 61v, ,Verteidigung', vgl. Sommer 1833:135); Replica (fol. 45v, ,die zweite Klageschrift, Antwort auf Einwürfe des Beklagten', vgl. Sommer 1833:422), Caution (fol. 48v); Juramentum (fol. 52r, ,Eid ,Eidschwur', Sommer 1833:266); Sentencia (fol. 52r, ,Rechtsspruch, Richterspruch, Urteil', Sommer 1833:447) oder euiction (fol. 50v, ,Gewährleistung, Bürgschaft', Sommer 1833:180). Mit dem Benehmen der Klienten während der Verhandlung hängt die In finuaci- 
on an den Celbigen Richter ader Foyt (fol. 20r, ,Einschmeichelung', Sommer 1833:255) zusammen, am Ende des Prozesses werden penitencz (fol. 66v, ,Buße', Sommer 1833:374), gerichtliche Expens (fol. 72r, ,Kosten“, Sommer 1833:185) bzw. Moderation (fol. 72r Moderatio expensarum ,Herabsetzung der Kosten', Sommer 1833:308) festgelegt, oder es kommt zum Arreltiren vmb vngerichte boele tahten (fol. 28v).

Zur Bezeichnung mündlicher oder schriftlicher Produkte einer Rechtshandlung dienen die Termini Contract (in leinem gehaltenen Contract; fol. 28v), Regifter (in meinen Regiftern; fol. 53v), preambulum (ire preambulum machen; fol. 56r); Relation (Item der Gerichts diehner fal widerumb Relation thuen; fol. 79r; Relation ,Bericht, Anzeige', Sommer 1833:418), addicion, allegaten (mit viele nutzlichen addicion vnd beftenndigen allegaten der befchriebenen recht bei der materien der artickel; fol. I, Vorrede; addicion ,Zusatz', allegaten, eine aus einem Buch oder einer Schrift angeführte Stelle, Berufung auf ein Gesetz', Sommer 1833:24), Nota (fol. 25v); primus decretus (Ex primo decreto Inneheldtt; fol. 25r; decretus ,Beschluss', Sommer 1833:134); Artickl und Practica (diefes Tehre nutzliche buch der Artickl vnd Practica der gerichtsleuffte; fol. I, Vorrede) oder Claufela (wie den diefelbe Claufula in Colchen verfchreibungen; fol. 28v, 42v; Clausel ,Einschränkung, Vorbehalt‘, Sommer 1833:91). Der Rechtsterminus Citation (,gerichtliche Vorladung', Sommer 1833:90) erscheint entweder mit deutscher oder mit lateinischer Endung (Citation, fol. 79r; Citierung, fol. 2v; diefe Citacio, fol. 56r).

Eine spezifische Gruppe stellen Verben lateinischer Herkunft dar, die mit dem Suffix französischer Herkunft -ieren versehen sind. Dieses Suffix, das seit dem 14. Jh. häufiger im Frnhd. vorkommt, erscheint in Polans Rechtsvokabular relativ häufig: in der lachen fort zu procediren (fol. 57v, 70v, 80r; procediren, verfahren', Sommer 1833:391); dorfon nicht appeliren kan (fol. 67r); wider die zeugen excipirt (fol. 61v, 56v; excipiren, einwenden, entgegnen', Sommer 1833:182); Iein widerpartt dorczu citiren loIfen (fol. 55r, 29r; citiren ,vorladen, vorfordern', Sommer 1833:90); interrumpirt die verjharunge (fol. 46v; interrumpiren, unterbrechen', Sommer 1833:259); nicht difputirt (fol. 45r; disputiren ,mit Worten streiten', Sommer 1833:152) oder nicht zugeloffen, fonder wirt repellirtt (fol. 41v; repelliren, verstoßen, zurücktreiben‘, Prätorius 1875:247).

Nur an zwei Stellen findet man direkt im Text eine explizite Erläuterung dieses Terminus bzw. einen Verweis auf seine deutsche oder lateinische Bedeutung: vnnd folche brieffe haift man Executoriales (fol. 71r; ,Vollziehungsbefehle‘, Sommer 1833:183); Die Caution aber ift nicht eine folche burgfchafft, ..., Condern es ift ein Colcher vorltand vnd burgfchafft (fol. 48r). Ebenso stellt Polan in zwei Fällen ein lateinisches Wort und sein deutsches Äquivalent nebeneinander ohne Verbindungselement: Finis. das ende (fol. 77v) und Duplica, widerrede (fol. 45v; Duplica, die zweite Verantwortungsschrift des Beklagten auf die Replik des Klägers, die auf die erste Verantwortungsschrift des Beklagten, Exception, gefolgt ist', Sommer 1833:159).

Es gibt noch weitere Möglichkeiten, wie Polan dem Leser lateinische Rechtstermini näherbringt. Er schreibt sie an den Rand, und im Text kommt die deutsche Beschreibung bzw. das deutsche Äquivalent vor. Als Beispiele können folgende Marginalbemerkungen dienen: Utilitas guarande (fol. 50v), im Text Von der Gewehre der Clage oder am Rande Petitio condemnationis in penam guarande (fol. 53r), im Text Wen die gewehre gerurt, wie beklagter fich halten Cal und das letzte Beispiel: am Rande Sentencia diffinitiua (fol. 64v) und im Text Vor dem endtvrteil.

Eine andere Möglichkeit, wie Polan einen lateinischen Terminus erklärt, stellt die Verbindung eines relativ verständlichen Ausdrucks und des entsprechenden lateinischen Rechtsterminus dar, der sich in Klammern befindet (fol. 61v): ein auffchliefender termin (Et sic terminus exclusivus). Diese Fälle kommen jedoch nur vereinzelt vor.

\subsection{Formeln mit lateinischen Komponenten}

$\mathrm{Zu}$ einem besseren Verständnis lateinischer Termini tragen sicher auch Paarformeln bei, in denen sich neben einem lateinischen Rechtsterminus auch sein deutsches Äquivalent befindet - entweder ein synonymer, oder ein bedeutungsähnlicher Ausdruck. Aus semantischer Sicht gehören diese Termini 
meistens in den Bereich der Rechtshandlungen, aber einige bezeichnen auch Gerichtspersonen: von

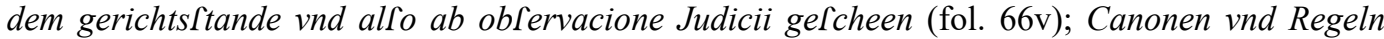
(fol. 66r); auff die belchuldigunge ader weißartickl (fol. 57r, wize ,Strafe'); Von den Interrogatorien vnd Fragftucken der Zeugen (fol. 57r); Andtwort ader Sententia (fol. 51v); eroeffnunge vnnd publicirunge der Zeugen Iage (fol. 60v); Arreftiren vnd auffhaldten (fol. 2v) oder Peen vnd Itraffe (fol. 53r). Adjektivische Attribute oder Genitivattribute können die Aussage weiter präzisieren: mit leibes Itraffe ader andern rechtmelligen penen (fol. 44r).

Eine Untergruppe bilden wieder Verben und Partizipien: verteilet [!] vnnd Condemnirt ift (fol. 71r; condemniren ,verurteilen“; Sommer 1833:104); von der fache difputirenn vnd redenn (fol. 40r; disputiren ,fechten, streiten“; Sommer 1833:152); proteftiret vnd zuvorbehalten hot (fol. 61r); nichts zuerfuellen ader zu fuplirenn (fol. 48v); von gethaner klage abfoluiren vnd ledigkh erkennen (fol. 2r) oder gefchicktt vnnd habilitirt lein (fol. 2r). Adjektive oder Adverbien kommen als Formeln dieses Typs nur sporadisch vor ( $k$ wit, ${ }^{37}$ loes vnd ledigk; fol. $7 \mathrm{v}$ ).

Einige lateinische Termini erscheinen im Text zunächst ohne Erklärung, erst später werden sie erläutert: Ceine exception ader Гchuczwern nicht abfchlohenn (fol. 45r), zuvor Ceine Exception getraue zubeweyfzenn (fol. 40r) ohne Erklärung.

Oft erscheinen bei denselben Rechtstermini unterschiedliche deutsche Äquivalente, die jedoch meistens Synonyme sind: die Expens vnnd gerichts koefte (fol. 49r); Expens vnd vncoltenn (fol. 72r) oder die Expens vnd Ichaden (fol. 48r), weitere Beispiele: diefe Citation ader befchickunge (fol. 24v); folche forderunge vnd Citacion (fol. 79r); Citirung vnd verkundigungkh (fol. 71r); die rechtliche Citirunge vnd ladunge gegen dem widertail (fol. 2v); Dan ein Jeder, der Cityrt vnd vor Recht erfordert wirt (fol. 45r); defenfion vnd Ichuczrede (fol. 61v) oder als letztes Beispiel die wehre vnd defenfion (fol. 45r).

Nur vereinzelt wird ein lateinischer Terminus mit Hilfe eines anderen lateinischen Ausdrucks erläutert, z. B. Vnd diefe Citacio ift endtlich vnd peremptorie (fol. 56r; peremptorie, die letzte Vorladung vor Gericht', Prätorius 1875:210). Ausnahmsweise stehen auch zwei lateinische Termini nebeneinander: Ein Jeder procurator ader aduocat (fol. $38 \mathrm{v}$ ); leinen parteyen vnd clienten (fol. 39v).

Manche lateinische Rechtstermini werden mit Hilfe einer Koppelung und außerdem noch anhand einer Definition erläutert, beispielsweise

Was Caution vnd vorftandt ift: Caution vnd vorftandtt ift bei rechte nichtes anders, den das der kleger vergwi fen mus, who ehr mit feiner klage vnd Cache felligk vnd verluftigkh erkandt wuerde (fol. 47r).

Was litis conteftadio / kriegs befeftigung bei rehte haift. Das heift bey rechte den kriegk befeltigenn, als wen der beklagte zur klage yha ader neyn laget (fol. 46r).

Was Citacion ift. Citation ader vorladunge ift ein felbftändiges thuen vnd der anfange des gerichts vnd gerichts Zwanges, vnd ein fundament des procels des gerichts (fol. $4 \mathrm{r}$ ).

Fälle, in denen zuerst ein deutscher Ausdruck und dann das lateinische Äquivalent angeführt sind, sind seltener: Es wird auch dem kleger noch gethaner anttwortt des beklagtenn nicht mehr dan nur eine rede, das ift eine replica, zugeloflen (fol. 44v).

Neben synonymen bzw. bedeutungsähnlichen Komponenten werden eher sporadisch auch Ausdrücke, die mehrere Seiten eines Sachverhalts nennen, gekoppelt: Von Inuentirung vnd $\Upsilon$ chatczung (fol. 20r); Areftiren vmb vngerichte boefe tahten, als todtfchlagkh, wunden, lehmnis, Iniurien etc. (fol. 28v). In der Gerichtsordnung sind daneben auch Ketten zu finden, die aus einem allgemeinen deutschen Ausdruck und einem lateinischen Terminus bestehen. Aus semantischer Sicht sind die Bezeichnungen von Personen im Gerichtsprozess am häufigsten vertreten: aus beuehl feines hern vnd principal (fol. 42v); zu einem advocaten oder vormunden (fol. 39r); zu einem procurator ader vormunden (fol. 80v), von Procuratorn, Aduocaten vnd Rednern (fol. $38 \mathrm{v})$.

37 Urspünglich vom lat. quietus, ins Deutsche aber aus dem altfranzösischen quite entlehnt. 
Die Untersuchungen bestätigen die Annahme, dass lateinische Rechtsbegriffe zu den wichtigsten Charakteristika des Vokabulars der Olmützer Gerichtsordnung gehören. Interessant sind ihre Verwendung und insbesondere die verschiedenen Möglichkeiten ihrer Erläuterung. Während das Rechtsvokabular in der Gerichtsordnung, das aus Erbwörtern besteht, seine Quelle im Meißner Rechtsbuch oder im Sachsenspiegel hatte, ergibt sich die Frage, wie Heinrich Polan zu seinen lateinischen Rechtstermini gekommen ist.

\section{Quellen der lateinischen Termini in der Olmützer Gerichtsordnung}

Wie Heinrich Polan im Titel seiner Gerichtsordnung andeutet, handelt es sich wahrscheinlich um zusammengetragene Artikel aus der Feder anderer Autoren oder Juristen. Die meisten Informationen wurden aus dem sächsisch-magdeburgischen bzw. Breslau-Magdeburger Schöffenrecht übernommen. Dem Brünner Rechtshistoriker Alfred Fischel zufolge ist

„[d]ie Bedeutung der Olmützer Gerichtsordnung [...] allerdings nicht in der Richtung zu suchen, ob es eine mehr oder minder wissenschaftliche Bearbeitung des damaligen Prozeßrechtes darstellt, sondern darin gelegen, daß in ihr getreu wie in einem Spiegel der gesamte Rechtsgang mit allen überlieferten und durch ihr hohes Alter und den nationalen Gehalt ehrwürdigen Förmlichkeiten des deutschen Rechtes für die späten Enkel zu schauen ist.“(Fischel 1903:XX)

Die Hauptquellen beim Verfassen der Gerichtsordnung waren für Polan das Werk Processus und Practica aus dem Jahre 1541 von Kilian König, das Werk Gerichtlicher Prozeß von Andreas Perneder (1545) und Perneders Übersetzung der Summa Rolandina des Soccinus aus dem Jahre 1544, von denen er für seine Texte viele Informationen übernahm, obwohl er sie namentlich nicht anführt. Er beruft sich aber auf den einflussreichen Juristen des 16. Jh. Ulrich Zasius und auf Henning Göde (bei Polan Goden genannt), von dessen Werk Iudiciarii ordinis processus aus dem Jahre 1538 Polan mehrere Stellen übernehmen sollte. Außerdem dürften manche Beschlüsse des Olmützer Stadtrates aus dem Jahre 1547 als Unterlagen beim Verfassen des Rechtsdokuments gedient haben (Fischel 1903:20-21).

In der folgenden Tabelle wird das Vorkommen der Rechtstermini lateinischer Herkunft ${ }^{38}$ in den Registern der Werke Königs, Perneders und in Polans Text verglichen.

\begin{tabular}{|l|l|l|l|}
\hline Rechtsterminus $^{\text {Kilian König (1541) }}$ & Andreas Perneder (1545) & Heinrich Polan (1550) \\
\hline Advocat $^{39}$ & - & Vom ampt der aduocaten & $\begin{array}{l}\text { Von Procuratorn, Aduoca- } \\
\text { ten vnd Rednern }\end{array}$ \\
\hline Appellirung $^{40}$ & $\begin{array}{l}\text { Von der Appellirunge des } \\
\text { Anwalden }\end{array}$ & Appellation & - \\
\hline Arbiter $^{41}$ & $\begin{array}{l}\text { Von dem Entfcheider oder } \\
\text { Arbitro }\end{array}$ & - & - \\
\hline Arbitrator $^{42}$ & $\begin{array}{l}\text { Von dem Gleicher oder } \\
\text { Arbitratore }\end{array}$ & - & - \\
\hline Attentata $^{43}$ & - & $\begin{array}{l}\text { Das die attentata zwayerlay } \\
\text { geftalt iuftificirt werden }\end{array}$ & - \\
\hline
\end{tabular}

38 Ein ausführlicher Vergleich dieser Werke im jeweiligen Forschungsprojekt wird für das nächste Jahr geplant.

39 Aduocat, Anwalt, Sachwalter".

40 Appelirung, Berufung auf ein höheres Gericht`.

41 Arbiter ,Schiedsmann".

42 Arbitrator,Schiedsmann".

43 Attentata, Eingriffe in fremde Rechte'. 


\begin{tabular}{|c|c|c|c|}
\hline Caution $^{44}$ & $\begin{array}{l}\text { Von den Vorftenden vnd } \\
\text { Caution }\end{array}$ & Caution & Caution ader vor_tandtt \\
\hline $\mathrm{Ce} \_$ion $^{45}$ & - & $\begin{array}{l}\text { Das die cerrion vnd } \\
\text { abtrettung der gFter den } \\
\text { Cchuldner nit volkommenlich } \\
\text { erledigen mogen }\end{array}$ & - \\
\hline Citation $^{46}$ & Von der Citation & Citation oder ladung & Citacio, Fuerladunge \\
\hline $\begin{array}{l}\text { Defenfor/ } \\
\text { DefenCion }^{47}\end{array}$ & $\begin{array}{l}\text { Von dem Vertretter oder } \\
\text { Defenfore }\end{array}$ & - & $\begin{array}{l}\text { Des Zeugenfuerers } \\
\text { Defenfion Ceiner Zeugen }\end{array}$ \\
\hline Dekret ${ }^{48}$ & - & Decret oder erkantnu $\beta$ & Ex primo decreto \\
\hline Delegat ${ }^{49}$ & $\begin{array}{l}\text { Von dem Befehlhaber } \\
\text { oder Delegaten }\end{array}$ & - & - \\
\hline Dilation $^{50}$ & $\begin{array}{l}\text { Von den Dilation vnd } \\
\text { Friftungen }\end{array}$ & Dilation & weyfaunge ader dilation \\
\hline Exception $^{51}$ & $\begin{array}{l}\text { Von den Wehren vnd } \\
\text { Exceptionibus }\end{array}$ & Einred. Exception & $\begin{array}{l}\text { Exception ader fchuc- } \\
\text { zwere }\end{array}$ \\
\hline Edict $^{52}$ & $\begin{array}{l}\text { Von der Citation per } \\
\text { Edictum }\end{array}$ & - & - \\
\hline Execution $^{53}$ & - & Execution oder volziehung & $\begin{array}{l}\text { das die huelffe vnnd } \\
\text { execution hernoch folge }\end{array}$ \\
\hline Iniurien $^{54}$ & $\begin{array}{l}\text { Von der Clage der } \\
\text { Iniurien vnd erftlich von } \\
\text { der wortlichen Iniuria }\end{array}$ & - & Iniurien \\
\hline Inftrument $t^{55}$ & $\begin{array}{l}\text { Von den Gezeugen der } \\
\text { Inftrumenten / Teftamen- } \\
\text { ten oder andern vrkunden }\end{array}$ & $\begin{array}{l}\text { So ain inftrument an zal der } \\
\text { zeugen mangelhafft wäre }\end{array}$ & rechtmeflige Inftrument \\
\hline Interrogatoria $^{56}$ & $\begin{array}{l}\text { Von den Interrogatorien } \\
\text { oder Fragftuecken der } \\
\text { Zeugen }\end{array}$ & - & $\begin{array}{l}\text { von den Interrogatorien } \\
\text { vnnd fragftucken }\end{array}$ \\
\hline Libell $^{57}$ & $\begin{array}{l}\text { Von der Clage oder Libell } \\
\text { an im KelbIt }\end{array}$ & $\begin{array}{l}\text { Libell oder fchrifftlich } \\
\text { Klagen }\end{array}$ & klage \\
\hline
\end{tabular}

\footnotetext{
Caution ,Bürgschaft, Gewährleistung،.

Ceffion, Abtretung einer Sache oder eines Rechtes an einen anderen`.

Citation, Vorladung vor Gericht'.

47 Defenfor / Defenfion, Verteidiger, Beschützer‘. Defen_ion, Verteidigung‘.

48 Dekret,Beschluss'.

49 Delegat, der Angewiesene (in Betreff einer Zahlung oder Schuld); der Abgeordnete‘.

50 Dilation, Verschiebung, Verzögerung, Frist".

${ }^{51}$ Exception, die erste Verantwortungsschrift des Beklagten“.

52 Edict,Erlass'.

53 Execution, Vollziehung‘.

54 Injuria, Injurie, Ehrverletzung, Schimpf- oder Schmährede, Beleidigung‘

55 Inftrument, Beweisurkunde'.

${ }_{56}$ Interrogatoria, gerichtliche Fragen, Verhör‘.

57 Libell,Klagschrift‘.
} 


\begin{tabular}{|c|c|c|c|}
\hline Notar & Von dem offen Notario & $\begin{array}{l}\text { Das ain Notari oder } \\
\text { gerichtfchreyber auch } \\
\text { reculiert werde }\end{array}$ & Notarienn \\
\hline Part/Parthey & $\begin{array}{l}\text { Von der erfcheinunge der } \\
\text { Part im Termin }\end{array}$ & Parthey & Parthen \\
\hline Procurator & $\begin{array}{l}\text { Von dem Anwalden oder } \\
\text { Procuratore }\end{array}$ & - & Procurator \\
\hline Prothocoll & Von dem Prothocollo & - & - \\
\hline Publication $^{58}$ & $\begin{array}{l}\text { Von der eroffenunge vnd } \\
\text { publication des Gezeugnis }\end{array}$ & - & $\begin{array}{l}\text { Von der eroeffnunge der } \\
\text { Zeugen lage }\end{array}$ \\
\hline Recu_atio ${ }^{59}$ & $\begin{array}{l}\text { Von der recufatio des } \\
\text { Richters }\end{array}$ & Recufation & - \\
\hline Repetitio & $\begin{array}{l}\text { Von der wider Verhörung } \\
\text { vnd allo de Repetitione } \\
\text { teltium }\end{array}$ & - & - \\
\hline Syndicus & Von dem sindico & $\begin{array}{l}\text { Von den Sindicis der ftet } \\
\text { vnnd commun }\end{array}$ & - \\
\hline Tranৎৎumpt ${ }^{60}$ & Von dem Tranৎlumpto & - & - \\
\hline
\end{tabular}

Tab. 1: Vergleich der ausgewählten Rechtstermini bei Kilian König, Andreas Perneder und Heinrich Polan

Es ist zu betonen, dass nur die Register in zwei Werken untersucht wurden. Von 28 Rechtstermini, die wenigstens in einem Register vorkommen, treten insgesamt sieben Rechtsausdrücke sowohl in den beiden Registern und in Polans Text auf - Caution, Citation, Dilation, Exception, Inftrument, Notar und Part/Parthey. In Königs Register und Polans Text treten fünf Rechtstermini auf - Defenfor/Defenfion, Iniurien, Interrogatoria, Procurator und Publication; in Perneders Register und Polans Text sind drei Rechtsausdrücke zu finden - Aduocat, Dekret und Execution.

Heinrich Polan übernahm Informationen und damit auch Rechtsausdrücke auf ganz unterschiedliche Weise - es gibt aber nur selten Texte in seiner Gerichtsordnung, die entweder von König oder von Perneder bloß abgeschrieben wurden. Polan ging mit den Informationen kreativ um. Wichtig war auch die Tatsache, dass dabei Rechtsausdrücke lateinischer Herkunft in die deutsche Rechtssprache entlehnt wurden. Bereits die Untersuchung beider Register zeigt, dass sowohl Practica von König als auch Der gerichtliche Process wichtige Quellen für Heinrich Polan waren. Einen Beweis dafür bietet uns der folgende Vergleich zweier ausgewählten Mikrotexte, die sich mit denselben Themen befassen:

\begin{tabular}{|c|c|}
\hline Heinrich Polan (1550) & Kilian König (1541) \\
\hline $\begin{array}{l}\text { fol. } 50 \mathrm{v} \\
2 \text { Die erfte woltadt yft diefe, das der kleger dem } \\
\text { beklagtenn die klage, dermoflenn gewehren mus, } \\
\text { Who ynen ein ander vmb diefe fachen ins recht zie- } \\
\text { hen vnd beklagenn woldte, das ehr Inen vertrehtenn } \\
\text { mus, vnd hot alfo die gewehre in diefem vhalle die } \\
\text { wirkunge der euiction. }{ }^{61}\end{array}$ & $\begin{array}{l}\text { Cap. XLIX } \\
\text { Die erfte das der Cleger dem beclagten die clage } \\
\text { dermaffen gewehren mus / wo jnen ein ander vmb } \\
\text { diefe fache ins recht zihen vnd beclagten wolte / } \\
\text { das er jn vertreten mus / Vnd hat alfo die gewehr in } \\
\text { diefem fall / die wirckunge der euiction. }\end{array}$ \\
\hline
\end{tabular}

$58 \quad$ Publication, Veröffentlichung‘.

59 Recufatio, Ablehnung, Verweigerung‘.

60 Tranfrumpt, eine beglaubigte Abschrift‘

61 Am rechten Rand dieses Mikrotextes ist geschrieben: Effectus guarande. Vt Landt li[ber] 2. art. 15. et li[ber] 3. art. 14. in tex. et glof. li[ber] 1. ar. 63. et li[ber] 2. art. 14. \$. wer da. Weich. ar. 23. in glof. col. 12. verfic. Ihr foldt wiffen et Weich. ar. 40. in glor. fina. 


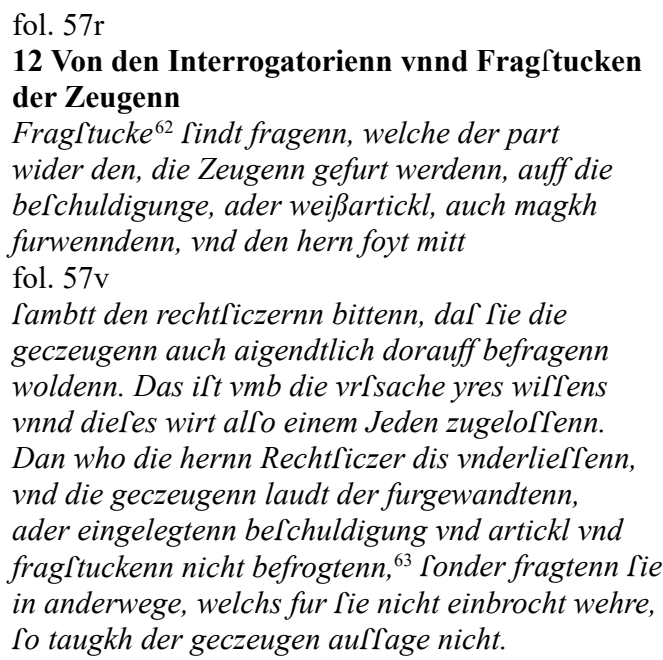

\section{Cap. LXXX}

Frageltucke find fragen / durch welche der teil wider den die gezeugen gefurt / die lelbigen auff die artickel vnd jre vmbltende wil fragen la斤Ten / ut in c. praesentium. Adde ...

Vnd wo der Richter in Teiner citation / darinn er den parth citirt / das er erfcheine / lehe vnd höre / die gezeugen fur zuftellen etc. vnd jm darbey die artickel zufendet / mit anzeigunge das er im termin leine frageltucke ob er der gebrauchen wolle leinlege etc. Io hat er die aus den artickeln zu machen vnd zuftellen / Denn wo er die lonlt auff befragt werden / Io werden lie nicht zugelarlen / Vnd Io die gezeugen darauff befragt werden / Io were jr auৎlage / als vnuereidet nichtig / de quo in c. de teltibus extra de telt.

Tab. 2: Vergleich der ausgewählten Mikrotexte bei Heinrich Polan und Kilian König

Nach welchen Kriterien Heinrich Polan Passagen über Rechtshandlungen und Rechtsgebrauch in seine Gerichtsordnung übernahm, ist nicht ganz klar. Selbstverständlich spielte vor allem die Tatsache eine wichtige Rolle, ob Rechtshandlungen in der Stadt Olmütz zu finden waren. Es gibt aber auch Begriffe, die für Olmütz wichtig waren, aber in der Olmützer Gerichtsordnung trotzdem nicht vorkommen, obwohl sie in Königs und in Perneders Werk zu finden waren, wie z. B. der Begriff syndicus, die Bezeichnung eines wichtigen Beamten. Eine kurze Passage über dieses Amt ist sowohl in Königs als auch in Perneders Werk zu finden, in Polans Gerichtsordnung fehlt jedoch dieses Kapitel. Es ist nicht einfach zu erklären, warum Polan das Amt des Syndicus theoretisch nicht behandelte, obwohl es in der Olmützer Stadtkanzlei ab Mitte des 15. Jh. existierte (Spáčil 2001:74). Der erste Olmützer Syndicus, der nicht anonym war, war David, Generalprokurator des Olmützer Konsistoriums, daneben ein öffentlicher Notarius. Im Zusammenhang mit diesem Amt erscheint im Jahre 1456 Davids Familienname Einsiedl (Spáčil 2001:74). Der Syndicus war ein diplomatischer Rechtsvertreter der Stadt, ein Rechtsberater des Stadtrates in komplizierten Rechtsverhandlungen. In der Mitte des 16. Jh. flossen das Amt des Syndicus und das Amt des Stadtschreibers in Olmütz in einem Amt zusammen. Wahrscheinlich dürfte das ein Grund dafür gewesen sein, warum Polan dieses Amt in seiner Gerichtsordnung nicht behandelte.

In allen drei Werken kann man Wortketten finden, in denen lateinische Rechtstermini dadurch semantisiert wurden, dass ein deutsches Äquivalent hinzugefügt oder eine Definition verwendet wurde: Procurator oder Vorspreche (König), Von den Interrogatorien oder Fragstuecken der Zeugen (König), Von den Wehren vnd Exceptionibus (König), Von der eroeffenunge vnd publication des Gezeugnis (König), Von der Clage oder Libell (König), Von den Vorftenden vnd Caution (König), Citation oder ladung (Perneder), von exception vnd einreden (Perneder), Decret oder erkantnu $\beta$ (Perneder), Execution oder volziehung (Perneder); ähnlich auch bei Polan, wie das oben behandelt wurde: Citacio, Fuerladunge, weyৎzunge ader dilation, Litis contestation ader kriegs beuestung, Caution ader vorftandtt.

62 Am linken Rand ist geschrieben: Propter duo enim inuenta funt huius modi interrogatoria, nidelicet ad deprehenfionem falfitatis et inftructionem iudicis siue recipientis teftes. Ita notat Joan. and. in cap. per tuas Extra de telti. in gloS. 1. Rechts ist geschrieben: Interrogatoria, Fragftuck. Sowohl der Anmerkung 60 und als auch dieser Anmerkung 61 ist zu entnehmen, dass lateinische Termini in Königs Werk eine wichtige Rolle spielten. Wenn dieses Werk dem Verfasser der Olmützer Gerichtsordnung als eine wichtige Informationsquelle diente, liegt auf der Hand, warum den lateinischen Termini in der Olmützer Gerichtsordnung eine so bedeutende Stelle zustand. 


\section{Schluss}

Die bisherigen Untersuchungen der Olmützer Gerichtsordnung führen zu folgendem Schluss:

Die Basis der Rechtsterminologie in der Olmützer Gerichtsordnung Heinrich Polans bilden die deutschen Erbwörter, die bereits im Sachsenspiegel oder im Meißner Rechtsbuch vorkommen. Die beiden Rechtsdokumente werden deswegen erwähnt, weil sie die Grundlage des sächsisch-magdeburgischen Rechts bilden. Das Olmützer Stadtrecht gehörte zu den Modifikationen dieses Rechts.

Die Rezeption des römischen Rechts führte nicht nur in den zentralen deutschen Schreiblandschaften, sondern auch in den Stadtkanzleien an der Peripherie, zu denen auch die Olmützer Stadtkanzlei zählt, zu einer weiteren Entfaltung der deutschen Rechtssprache. Eines der wichtigsten Merkmale des Vokabulars der Olmützer Gerichtsordnung sind lateinische Termini, die entweder in Form von Paraphrasierungen, Definitionen oder deutschen Äquivalenten erklärt wurden oder in Formeln mit interpretierenden Synonymen oder bedeutungsähnlichen Ausdrücken vorkommen. Gerade die Semantisierung lateinischer Rechtstermini wird von Polan meisterhaft beherrscht. Ziel des Verfassers war es, die Verständlichkeit einzelner Artikel in der Gerichtsordnung zu garantieren. Die benutzten Paarformeln, die aus deutschen Begriffen bestehen, sollten eine präzise Ausdrucksweise und eindeutige Informationen über Grundsätze bezwecken, die beim Verlauf des Gerichtsprozesses akzeptiert werden müssen. Im Unterschied zu den Formeln mit lateinischen Termini war ihr Vorkommen in der Gerichtsordnung nicht so häufig.

Die Untersuchung der Lehnwörter in der Olmützer Gerichtsordnung hat gezeigt, dass Latein im Zeitraum vom 15. bis 17. Jh. die wichtigste Sprache war, aus der sich das frnhd. Rechtsvokabular konstituierte. Auch die Schreiber konnten die Durchsetzung der neuen Rechtsterminologie durch ihre Latein- und Rechtskenntnisse beeinflussen. Ihre Kompetenz erlaubte ihnen, lateinische Termini zu benutzen, die dann von ihren Nachfolgern übernommen wurden, so dass sich am Ende des 16. Jh. ein Kanzleiusus herausbildete, in dem Ausdrücke lateinischer Herkunft große Bedeutung hatten. Der Gebrauch der lateinischen Rechtsterminologie war im letzten Viertel des 16. und im 17. Jh. intensiv; es kam sogar zum Ersatz deutscher Endungen bei eingebürgerten Lehnwörtern durch lateinische. Dies ließe sich damit erklären, dass der exklusive Rechtswortschatz einschließlich der lateinischen Endungen einen besseren Eindruck machen sollte. In der Olmützer Gerichtsordnung finden wir hierfür mehrere Beispiele.

\section{Literaturverzeichnis}

Prmärliteratur:

ASUC $=$ Album studiosorum universitatis Cracoviensis. Band 2 (ab anno 1490 ad annum 1551). Herausgegeben von Chmiel, Adam. Kraków 1892.

Die Bibel nach der Übersetzung Martin Luthers. 4., revidierte Fassung. Berlin; Altenburg 1990.

Fischel $(1903)=$ Die Olmützer Gerichtsordnung. Ein Beitrag zur Geschichte des österreichischen Prozessrechtes. Herausgegeben von Fischel, Alfred. Brünn 1903.

Hass-Zumkehr, Ulrike (1986): Leonhard Schwartzenbachs Synonyme. Beschreibung und Nachdruck der Ausgabe Frankfurt 1564. Tübingen.

Kodex Wenzels von Iglau, Landesarchiv Opava, Zweigstelle Staatliches Bezirksarchiv Olomouc, Bestand Archiv der Stadt Olmütz, Bücher, Sign. 1540.

KöNIG, Kilian: Processes und Practica der Gerichtsleuffte nach Gebrauch Sechsischer Landart. [Leipzig] 1541.

KWI = SPÁČILOVÁ, Libuše - SpáčIL, Vladimír (2004): Památná kniha olomoucká (kodex Václava z Jihlavy) z let 1430-1492, 1528. Úvod, jazykový rozbor německých textů, edice, rejstř́ky. Olomouc.

MRB = Das Meißner Rechtsbuch. Historischer Kontext, linguistische Analyse, Edition. Herausgegeben von SPÁčIL, Vladimír und SpáčILová, Libuše. Olomouc 2010.

MUNWU = Die Matrikel der ungarischen Nation an der Wiener Universität 1453-1630. Herausgegeben von Schrauf, Karl. Wien 1902. 
Polan, Heinrich: Ratesspiegel, Landesarchiv Opava, Zweigstelle Staatliches Bezirksarchiv Opava, Bestand Archiv der Stadt Troppau, Bücher, Sign. 221.

$\mathrm{SSp}=$ Eike von Repgow: Der Sachsenspiegel. Aus dem Mittelniederdeutschen übersetzt von SchmidT-Wiegand, Ruth und Schотт, Clausdieter. Zürich 2006.

Stadtbücher der Stadt Olmütz, Landesarchiv Opava, Zweigstelle Staatliches Bezirksarchiv Olomouc, Bestand Archiv der Stadt Olmütz, Bücher, Sign. 2, 1046, 1048.

\section{Sekundärliteratur:}

Besch, Werner (1964): Zweigliedriger Ausdruck in der deutschen Prosa des 15. Jahrhunderts. In: Neuphilologische Mitteilungen 65, S. 200-221.

DILCHER, Gerhard (1961): Paarformeln in der Rechtssprache des frühen Mittelalters. Frankfurt a. M.

DinZELBACHER, Peter (1992, Hrsg.): Sachwörterbuch Mediävistik. Stuttgart.

DRW I/1914 = Deutsches Rechtswörterbuch. Wörterbuch der älteren deutschen Rechtssprache. Band I. Weimar 1914.

Habermann, Mechtild (2001): Deutsche Fachtexte der frühen Neuzeit. Berlin; New York.

Hartweg, Fréderic / Wegera, Klaus-Peter (2005): Frühneuhochdeutsch. Tübingen.

HonzÁk, František / NeŠKudla, Bořek / PeČEnKA, Marek / STELlner, František / VlčKová, Jitka (1997): Evropa v proménách staletí. Praha.

Jesko, Friedrich (2006): Phraseologisches Wörterbuch des Mittelhochdeutschen. Tübingen.

KeLLER, Rudolf E. (1995): Die deutsche Sprache. Hamburg.

Kluge. Etymologisches Wörterbuch der deutschen Sprache. 24. Auflage. Berlin; New York 2002.

KöBLER, Gerhard (2005): Zielwörterbuch europäischer Rechtsgeschichte. 3. Auflage. Gießen-Lahn.

Linke, Angelika / Burger, Harald (1998): Historische Phraseologie. In: Besch, Werner / BetTen, Anne / Reichmann, Oskar / Sonderegger, Stefan (Hrsg.): Sprachgeschichte. Ein Handbuch zur Geschichte der deutschen Sprache und ihrer Erforschung. 1. Teilband. Berlin; New York, S. 743-755.

MaLÝ, Karel (1995): České právo v minulosti. Praha.

MATZINGER-PFISTER, Regula (1972): Paarformeln, Synonymik und zweisprachiges Wortpaar. Zur mehrgliedrigen Ausdrucksweise der mittelalterlichen Urkundensprache. Zürich.

MerK, Walther (1933): Werdegang und Wandlungen der deutschen Rechtssprache. Marburg.

MitTEIS, Heinrich (1992): Deutsche Rechtsgeschichte. 19., neu bearbeitete Auflage von Lieberich, Heinz. München.

NiEDERSTÄTTER, Alois (1985): Vorarlberger Urfehdebriefe bis zum Ende des 16. Jahrhunderts. Eine Quellensammlung zur Rechts- und Sozialgeschichte des Landes. Dornbirn.

NowaK, Elisabeth (1965): Die Verbreitung und Anwendung des Sachsenspiegels nach den überlieferten Handschriften. Dissertation zur Erlangung der Doktorwürde der Philosophischen Fakultät der Universität Hamburg. Hamburg.

PAPSONOvá, Mária (2000): Zu semantischen Besonderheiten der deutschen Rechtssprache am Beispiel des Silleiner Rechtskodexes. In: brücken. Neue Folge 7, 1999, S. 243-259.

PfEIFER, Wolfgang et al. (1989): Etymologisches Wörterbuch des Deutschen. 3 Bände. Berlin.

PRÄTORIUS, Gregor (1875): Der Wortgrübler. Wien.

SCHMID, Hans Ulrich (2015): Historische deutsche Fachsprachen. Von den Anfängen bis zum Beginn der Neuzeit. Eine Einführung. Berlin.

SchmidT-Wiegand, Ruth (1984): Paarformeln. In: ErLer, Adalbert / KaUfManN, Ekkehard (Hrsg.): Handwörterbuch zur deutschen Rechtsgeschichte. Bd. 3. Berlin, Sp. 1387-1399.

SCHMIDT-WIEGAND, Ruth (1990): Rechtssprache. In: ErLER, Adalbert/KaUfManN, Ekkehard(Hrsg.): Handwörterbuch zur deutschen Rechtsgeschichte. Bd. 4. Berlin, Sp. 343-360.

SCHMIDT-WiEgand, Ruth (1998): Anwendungsmöglichkeiten und bisherige Anwendung von philologisch-historischen Methoden bei der Erforschung der älteren Rechtssprache. In: HofFMANN, Lothar / KALVERKÄMPER, Hartwig / WiEgAND, Herbert Ernst (Hrsg.): Fachsprachen: ein inter- 
nationales Handbuch zur Fachsprachenforschung und Terminologiewissenschaft. Halbband 1. Berlin; New York, S. 277-283.

SCHMIDT-WIEGAND, Ruth (1999): Der Rechtswortschatz im Sachsenspiegel. In: HofFMAnN, Lothar / KALVERKäMPER, Hartwig / WIEGAND, Herbert Ernst (Hrsg.): Fachsprachen: ein internationales Handbuch zur Fachsprachenforschung und Terminologiewissenschaft. Halbbd. 2. Berlin; New York, S. 2 341-2 348.

SCHMIDT-WIEGAND, Ruth (2002): Deutsche Rechtsregeln und Rechtssprichwörter. Ein Lexikon. München.

Schmidt-Wiegand, Ruth (1977): Eid und Gelöbnis, Formel und Formular im mittelalterlichen Recht. In: Classen, Peter (Hrsg.): Recht und Schrift im Mittelalter. Sigmaringen, S. 55-90.

Schмiтt, Ludwig Erich (1936): Die deutsche Urkundensprache in der Kanzlei Kaiser Karls IV. (1346-1378). Halle/Saale.

SOMmER, Johann Gottfried (1833): Neuestes wort- und sacherklärendes Verteutschungs-Wörterbuch aller jener aus fremden Sprachen entlehnten Wörter, Ausdrücke und Redensarten, welche die Teutschen bis jetzt, in Schriften und Büchern sowohl als in der Umgangssprache, noch immer für unentbehrlich und unersetzlich gehalten haben. Ein Handbuch für Geschäftsmänner, Zeitungsleser und alle gebildete Menschen überhaupt. Prag.

SpáčIL, Vladimír (2001): Písaři a kanceláre města Olomouce do roku 1786. Olomouc.

SpÁČILOvá, Libuše (2004): Rechtsterminologie lateinischer Herkunft in frühneuhochdeutschen Texten der Olmützer Stadtkanzlei. In: Germanoslavica 15, Nr. 2, S. 199-212.

SpáčILOvá, Libuše (2004): Zum Vokabular der Olmützer Gerichtsordnung aus dem Jahre 1550. In: BoKová, Hildegard (Hrsg.): Zur Erforschung des Frühneuhochdeutschen in Böhmen, Mähren und der Slowakei. Schriften zur diachronen Sprachwissenschaft. Wien, S. 171-192.

SpáčIlová, Libuše (2007): Die Olmützer Gerichtsordnung von Heinrich Polan aus dem Jahre 1550 als Textsorte. Ein Beitrag zur Untersuchung frühneuhochdeutscher Rechtstexte. In: Germanoslavica 18, Nr. 1-2, S. 49-61.

SpÁČILOvÁ, Libuše (2009): Der Olmützer „liber causarum criminalium“ als Quelle zur Untersuchung der frühneuhochdeutschen Rechtssprache. In: ERnst, Peter (Hrsg.): Kanzleistil: Entwicklung, Form, Funktion. Wien, S. 179-196.

SpáčILOvá, Libuše (2010): Die Textsorte Urfehde im Olmützer ,liber causarum criminalium“ aus den Jahren 1584-1629. In: Moulin, Claudine / Ravida, Fausto / Ruge, Nikolaus (Hrsg.): Sprache in der Stadt. Heidelberg, S. 223-242.

SpáčIlová, Libuše (2013): Das Fachvokabular im Meißner Rechtsbuch. In: WaGnerová, Marina / SANDNER, Gerg (Hrsg.): Die Rechtssprache in der internationalen Diskussion. Hamburg, S. $175-199$.

SpÁČILOvá, Libuše (2016): Vom Meißner Rechtsbuch zur Gerichtsordnung. Zur Entwicklung der Gerichtsrhetorik. In: HüNECKE, Rainer / AEHnelt, Sandra (Hrsg.): Kanzlei und Sprachkultur. Wien, S. 163-182.

Staehelin, Ernst (1955): Amandus Polan von Polansdorf. Studien zur Geschichte der Wissenschaften in Basel. Basel.

Štěpán, Václav (2000): Der Troppauer Stadtschreiber Heinrich Polan von Polansdorf und sein Sohn Amand. In: KoselLeK, Gerhard (Hrsg.): Oberschlesische Dichter und Gelehrte vom Humanismus bis zum Barock. Bielefeld, S. 247-253.

ZuKal, Josef (1927): Polanové z Polansdorfu. Památná rodina opavská 16. věku. In: Časopis Matice moravské 51, S. 99-123.

\section{Internetquellen:}

URL 1: Deutsche Wörterbücher. http://woerterbuchnetz.de [05.08.2020].

URL 2: Deutsches Rechtswörterbuch. http://drw-www.adw.uni-heidelberg.de/drw/ [08.08.2020].

URL 3: Frühneuhochdeutsches Wörterbuch. https://fwb-online.de [08.08.2020]. 